\title{
1 GAI MoRFs Regulate Cleft and Channel Binding Pathways for Gibberellin in GID1A
}

2 John Patterson ${ }^{1}$, Charles C. David ${ }^{2}$, Marion Wood $^{3}$, Xiaolin Sun ${ }^{3}$, Donald J. Jacobs ${ }^{1 *}$, Erik H. A.

3 Rikkerink $^{3 *}$

$4{ }^{1}$ Department of Bioinformatics and Genomics \& Department of Physics and Optical Science,

5 University of North Carolina at Charlotte, NC, USA

$6 \quad 2$ The New Zealand Institute for Plant and Food Research Ltd, Private Bag 4704

7 Christchurch Mail Centre, Christchurch 8140, New Zealand

$8{ }^{3}$ The New Zealand Institute for Plant and Food Research Ltd, Private Bag 92169, Auckland

9 Mail Centre, Auckland 1142, New Zealand

$10 *$ Corresponding authors

11 Graphical Abstract

13

14

15

16

17

19

18 (GID1) receptors to regulate DELLA degradation. The binding mechanism for GA was

20 family member GAI (GA Insensitive transcriptional repressor) from the GID1A receptor in the

21 presence and absence of bound GA, and of GA from GID1A in the presence and absence of

\section{Abstract}

The hormone gibberellin (GA) promotes arabidopsis growth

by enhancing binding between

GA Insensitive DELLA

transcriptional repressors and

GA Insensitive Dwarf 1

elucidated by employing a computational study of dissociations of the N-terminus of the DELLA 
22 GAI. The tRAMD method was employed to deduce egression pathways for a diverse set of GA

23 molecules $\left(\mathrm{GA}^{(\mathrm{x})}\right)$. Two pathways in the form of a newly identified cleft and a previously

24 identified channel are prevalent. The cleft pathway is open in the absence of GAI. Upon GAI

25 binding, the cleft route is blocked, resulting in a slower process for $\mathrm{GA}^{(\mathrm{x})}$ to exit and enter the

26 binding pocket through the channel. Several binding pocket residues are identified as gate-

27 keepers to the channel. Molecular recognition features (MoRFs) found in the disordered

28 signaling protein GAI affect $\mathrm{GA}^{(\mathrm{x})}$ binding and GID1A dynamics. A three-step synergistic

29 binding cycle is proposed where GAI MoRFs regulate the process. Rapid binding takes place

30 through the cleft where little to no distinctions are made between major and less active forms of

$31 \mathrm{GA}^{(\mathrm{x})}$. After GAI is bound to the $\mathrm{GA}^{(\mathrm{x})} \cdot$ GID1A complex, the channel supports a rectification

32 process that increases the retention of major active forms of GA within the binding pocket. Both

33 the cleft and channel contact residues to $\mathrm{GA}^{(\mathrm{x})}$ are markedly conserved in a GID1 phylogeny,

34 suggesting this binding process in the GID1 - DELLA GA-receptor complex represents a general

35 paradigm for GA binding. Non-specific GA binding assists binding of GAI, which then helps to

36 select the major active forms of the hormone and induce a downstream signalling cascade in

37 response to bioactive GA.

38 Non-expert Summary Statement

39 Gibberellins are plant hormones essential for growth and development. The DELLA proteins are

40 a disordered family of repressors that transcriptionally repress GA responsive genes. Degradation

41 of DELLA proteins in response to GA results in GA-responsive genes being upregulated.

42 Binding of GA to the GA-Insensitive Dwarf 1 receptor (GID1) facilitates binding of DELLA to

43 the GA - GID1 complex. Through computational modelling and phylogenetic analyses, we

44 identified a new GA binding cleft that is blocked by DELLA binding and a three-step 
45 mechanism for the GA $\cdot$ DELLA $\cdot$ GID1 complex that also involves the known GA binding

46 channel. We propose a dual (cleft/channel) pathway that allows access to the binding pocket as a

47 paradigm for selection of specific GA forms among a mixture of major active and inactive forms.

48 The cleft is less selective, but preference for active GA in the binding pocket of GID1A is

49 amplified by expunging inactive GA forms, followed by recruiting active forms through the

50 more selective channel. This mechanism allows plants to sense concentration changes of GA

51 with high specificity to enable certain GA variants to trigger specific signalling events. These

52 novel insights into the receptor mechanism in part may explain the large number of different GA

53 forms that exist in nature.

\section{Introduction}

55 It is well known that protein function does not only arise from highly structured parts of proteins

56 but also depends on regions that have flexibility (1-3). Furthermore, there is now general

57 recognition that highly flexible intrinsically disordered regions found in many proteins can be

58 critical for function. Disordered regions typically contain short semi-rigid segments that appear

59 to play key roles in establishing and controlling interactions with protein partners. Commonly

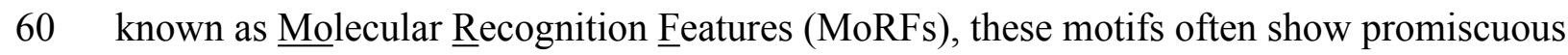

61 folding and binding activities that are enabled by their environments $(4,5)$. The large number of

62 accessible conformations in disordered proteins allows for binding promiscuity and/or a fine

63 gradient of responses. The DELLA protein GAI, one of 5 members in Arabidopsis, was first

64 identified through its function as a growth-repressing regulatory protein that operates through the

65 gibberellin response pathway (6). Subsequently the DELLA family was identified as a key site of

66 mutations in several grass species that have led to the so called 'Green Revolution' of high-

67 yielding dwarfed varieties. The dwarf phenotype stems from interruption of the interaction 
between the Gibberellin (GA) receptor GA-Insensitive Dwarf1 (GID1) and a DELLA protein

69 (7). Over the next two decades the DELLA family was shown to interact with a large group of

70 protein cohorts involved in a myriad of processes such as stress response (8), plant defence (9-

71 11), jasmonate signalling $(12,13)$, light perception mechanisms $(14,15)$, photosynthesis $(16)$,

72 stomatal conductance (17), circadian clock mechanisms (18), nitrogen assimilation (19), iron

73 uptake (20) and protein trafficking (21). The range of signalling pathways interacting with

74 DELLA proteins now covers virtually the entire suite of plant hormones $(22,23)$, earning them

75 the title of "master regulators of growth and development" (24).

76 The diversity of roles carried by this relatively small plant protein family is predicated upon its

77 structural plasticity and two main components. The structurally more rigid C-terminal GRAS

78 (Gibberellic-Acid Insensitive, Repressor of GAI and Scarecrow) domain is able to bind to many

79 transcriptional regulatory partners. The intrinsically disordered N-terminal domain contains the

80 family's eponymous conserved DELLA motif. We have previously proposed that the disordered

81 nature of the N-terminal domain, along with three short conserved motifs that act as MoRFs, are

82 critical to promote its hub function capabilities $(4,5,25)$. While these MoRF sites are known,

83 their intrinsic role in molecular recognition is incompletely characterized. Elucidating the

84 molecular details that describe how the DELLA protein interacts with the GID1 protein family

85 will help us understand how this protein complex can play such a key role in plant food

86 production and facilitate any future manipulation of this complex.

87 A GID1A centric hypothesis for the GA binding mechanism was initially developed based on the

88 GA - GAI - GID1A complex (gDG- where g refers to the hormone GA and D to the DELLA

89 protein GAI) that was crystalized (PDB-code: $2 \mathrm{ZSH}$ ). This proposed mechanism postulated a

90 large-scale motion in the flexible N-terminal region of GID1A that was thought to act as a lid 
91 that opens and closes onto the (previously) enzymatic core of GID1A $(26,27)$. Upon GA binding

92 to this open lid state of GID1A, the lid was proposed to subsequently close to form a cap. This

93 capping was deemed a necessary step to allow the GAI (DELLA) protein to bind, while re-

94 enforcing interactions between GA and the N-terminal extension of GID1A to maintain a stable

95 gDG complex. This sequence of events involving the intrinsic conformational motions of GID1A

96 and GA binding was proposed to create a recognition site for GAI. Once the GAI protein is

97 bound to the GA - GID1A complex to form gDG, it attracts a key component of the

98 polyubiquitination system and is subsequently degraded by the ubiquitin-mediated proteasomal

99 process. A consequence of this proposed mechanism is that once GAI is bound, the capped GID1

100 lid remains closed, preventing GA from leaving the binding pocket until the GAI protein is no

101 longer attached, typically as a result of proteolytic degradation.

102 The proposed capping of the GID1 N-terminal lid by DELLA proteins was challenged by Hao et

103 al. (28) through molecular modelling of the gDG complex. Without the GID1 "lid" opening up,

104 it was shown that there is sufficient space for GA to enter and exit the binding pocket through a

105 channel that is present in the GAI - GID1A complex. The free energy of binding through this

106 channel was calculated, and key contact residues within the channel were identified. These

107 results imply that an apo DELLA - GID1 complex (aDG) can exist within the cellular

108 environment. Interestingly, Yamamoto et al. (29) experimentally found that by suitable site

109 directed mutations to GID1, GA independent binding of DELLA proteins to the rice GID1 is

110 possible, albeit binding is still stronger in the presence of GA. They also identified weak binding

111 of arabidopsis GID1B and GAI in the absence of GA. Although there is no direct evidence that

112 GA independent DELLA - GID1 binding plays a regulatory role in plant biology, our modelling 
113 and analysis suggests that the aDG complex plays an important function as a kinetic intermediate

114 to ensure that the gDG complex contains a bioactive GA.

115 There is considerable interest in plant biology to better understand the roles of more than 100

116 variants of GA found in nature (30). The ability of different forms of GA to be converted to other

117 forms through a series of differentially regulated enzymatic steps, including both activation and

118 inactivation steps, makes it difficult to conclusively link bioactivity to the different forms of GA

119 without mutants in key enzymatic steps. For example, is it that some GA variants are transient

120 intermediate states or do they add to a spectrum of biological activity in their own right? The

121 large number of GA variants suggests the possibility of variable specificity for the receptor

122 and/or receptor complex. This high diversity motivated this study to consider distinctly different

$123 \mathrm{GA}^{(\mathrm{x})}$ on the binding mechanisms of DELLA to GID1A and GA ${ }^{(\mathrm{x})}$ to GID1A and the potential

124 implications of these steps to arrive at the gDG complex. A fundamental question is how

125 different GA variants bind to GID1 and comparatively, how effective they are in promoting the

$126 \mathrm{gDG}$ complex stability required for downstream proteolysis processes.

127 To glean insight into the role that $\mathrm{GA}^{(\mathrm{x})}$ ligand variants play, we investigated the $\mathrm{GA}^{(\mathrm{x})} \cdot \mathrm{GAI}$.

128 GID1A complex using the tRAMD computational method (31). Starting with a bound $\mathrm{GA}^{(\mathrm{x})}$ in

129 the binding pocket, this accelerated molecular dynamics approach was used to discover egression

130 pathways that enable $\mathrm{GA}^{(\mathrm{x})}$ dissociation. In addition to comparing different $\mathrm{GA}^{(\mathrm{x})}$ molecules, the

131 role of the DELLA protein MoRFs in the binding of GAI to GID1A in the presence and absence

132 of $\mathrm{GA}^{(\mathrm{x})}$ is assessed. Moreover, the dissociation of $\mathrm{GA}^{(\mathrm{x})}$ in the absence of GAI is investigated

133 and assessed to deduce the binding cycle that leads to the GA(x) GAI $\cdot$ GID1A complex. 


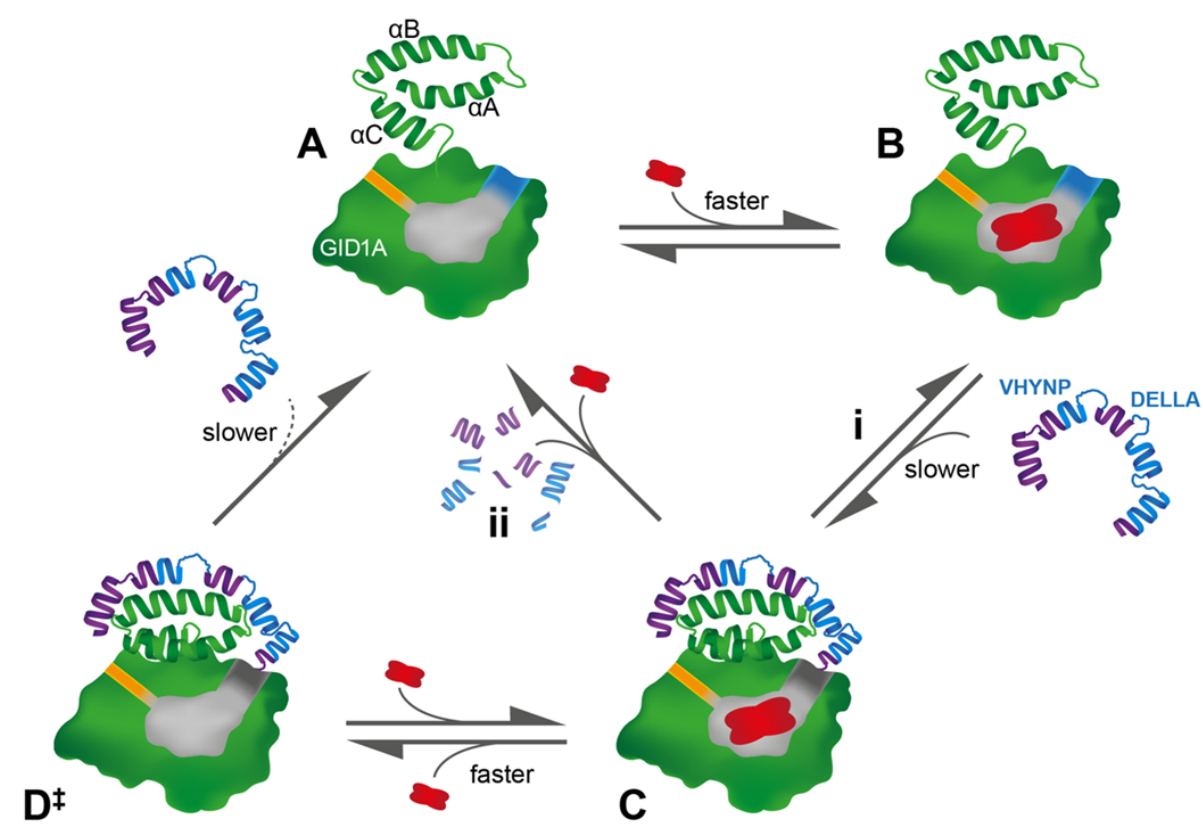

\begin{tabular}{|c|c|c|c|}
\hline Cleft pathway & Channel pathway & & GAI \\
\hline \multirow{2}{*}{$\begin{array}{l}\text { DELLA-induced Blocked } \\
\text { Cleft pathway }\end{array}$} & Binding pocket & $\begin{array}{l}\text { DELLA } \\
\text { VHYNP }\end{array}$ & $\begin{array}{l}\text { Molecular Recognition } \\
\text { Feature - MoRF }\end{array}$ \\
\hline & Gibberellin & & $\begin{array}{l}\text { Ubiquitin-mediated } \\
\text { proteolysis of GAI }\end{array}$ \\
\hline
\end{tabular}

Figure 1: Schematic of the computationally deduced GA binding process. (A) The GID1A receptor has two major routes of GA binding, a readily accessible newly identified cleft (blue) and a long channel (yellow). The GID1A cap ( $\alpha$-helices $A$ to $C$ ) is

instrumental in defining the binding pocket and it exhibits small-scale motions during the GA binding process. (B) After moving

138 through the cleft or channel, GA binds within the binding pocket in absence of GAI. (C). Upon binding of GAl, the DELLA MORF

139 covers the cleft access route for GA entry. The GA · DELLA · GID1A complex provides opportunity for degradation of GAI to occur

140 (ii). This can also result in a bound GA · GIDIA (i). (D) Due to large differences in time scales, the DELLA · GIDIA complex

141 facilitates GA binding through the channel before GAl dissociation occurs. D‡ indicates an unstable intermediate.

142 All modelling in this work considers the GID1A conformation with a closed lid. This closed state

143 is supported by the highly unfavourable free energy profile of the open "lid" (28) and the fact

144 that this is the only conformation with physical evidence from crystalisation. A prominent $\mathrm{GA}^{(\mathrm{x})}$

145 binding pathway through an open cleft in GID1A when GAI is absent is uncovered. The roles of

146 the MoRFs, residues that correspond to conserved motifs which are deleted in dwarf mutants of 
147 the DELLA partners, are identified with respect to these pathways. Conceptualized in Figure 1, a

148 complete kinetic cycle is proposed that provides a mechanism for preferential binding of major

149 bioactive GA in the receptor complex. This model provides significant new insights that explain

150 how specific GA regulation can occur within the presence of a large number of GA variants that

151 exist in nature. These insights will also inform strategies to manipulate the protein complex to

152 increase yield without negatively affecting the multitude of plant phenotypes linked to the

153 promiscuous DELLA family.

154 Results

155 GA variants considered

156 Among more than 100 different GA forms found in plants, the dichotomy of active and inactive

157 variants introduced early on (32) is likely too simplistic given the difficulty of tracking which

158 GA variant creates the signalling cascade after a particular form of GA is supplied exogenously.

159 To cover the spectrum of activities that may be inherent in this binary classification scheme, we

160 selected a diverse set of $\mathrm{GA}^{(\mathrm{x})}$ that includes GA variants widely referred to as bioactive $\left\{\mathrm{GA}^{(\mathrm{a})}\right\}$

161 and as inactive $\left\{\mathrm{GA}^{(\mathrm{i})}\right\}$. Additional types of GA are also considered to increase the diversity in

162 molecular moieties. Known $\mathrm{GA}^{(\mathrm{a})} \mathrm{GA}_{1}, \mathrm{GA}_{4}, \mathrm{GA}_{3}, \mathrm{GA}_{7}$, and recently reported $\mathrm{GA}_{12-16 \mathrm{x}}(33)$

163 were chosen to assess active GA containing systems. We also analysed a set of putative inactive

164 forms $\left(\mathrm{GA}^{(\mathrm{i})}\right)$ that represent variants with no evidence of biological activity. Most of these were

165 chosen as a progressive step from $\mathrm{GA}_{12}$, the main precursor to all $\mathrm{GA}$, to the various $\mathrm{GA}^{(\mathrm{a})}$

166 examined. In particular, $\mathrm{GA}_{4 \mathrm{MeO}}, \mathrm{GA}_{4-16 / 17 \mathrm{ox}}$, and $\mathrm{GA}_{34}$ are all oxidation products of $\mathrm{GA}^{(\mathrm{a})}$ and

167 are of interest to understand the signalling control in this system. 
169 All production runs for each $\mathrm{gDG}$ system retained the bound $\mathrm{GA}^{(\mathrm{x})}$ ligand within the binding 170 pocket. Counts of the recurrent hydrogen bonds (H-bonds) and hydrophobic contacts between

171 GA variants and the GID1A residues were monitored. Table 1 summarizes the binding pocking

172 interactions to $\mathrm{GA}^{(\mathrm{x})}$ that are largely in common across all the GA variants investigated here.

173 Highlighting these pocket residues outlines the proximity to key parts of GA 3 . TYR247 (see

174 methods for residue numbering) interacts with $\mathrm{GA}^{(\mathrm{x})} \mathrm{C} 3-\mathrm{OH}$, SER116 and GLY115 wrap around

175 the $\mathrm{C} 7$ carboxylate. TYR31 has interactions both with $\mathrm{OH}$ groups in those $\mathrm{GA}^{(\mathrm{x})}$ that have this

176 moiety around $\mathrm{C} 13$ and interactions with all GA vicinal C16-C17 bonds, appearing to be aided

177 by pi-pi interactions. This interaction aids in aligning GA inside the GID1A pocket and is a

178 shared moiety across most GA.

179 In the $\mathrm{GA}^{(\mathrm{x})} \cdot \mathrm{GID} 1(\mathrm{gG})$ systems a subset of common interactions was found. With no additional

180 interactions identified, the gG system maintains the same set of interactions except for

181 interactions with TYR31 and TYR247. The loss of the TYR31 and TYR247 interactions enable

182 there to be a less compact environment among the surrounding residues in gG systems compared

183 to gDG systems. Interestingly, the contact residues shown here are highly conserved across the

184 phylogeny of GID1 across diverse plant families (Tables S3-5) suggesting these residues have

185 similar significance beyond the Arabidopsis GAI - GID1A example that we analyze herein.

Table 1: Binding pocket recurrent hydrogen bonds, in blue, and hydrophobic contact residues, in black, from production runs of 


\begin{tabular}{|l|c|c|c|c|c|c|c|c|c|c|c|c|}
\hline RES & & & & & & & & & & & & \\
\hline ILE24 & $\checkmark$ & $\checkmark$ & $\checkmark$ & $\checkmark$ & $\checkmark$ & $\checkmark$ & $\checkmark$ & $\checkmark$ & $\checkmark$ & $\checkmark$ & $\checkmark$ & $\checkmark$ \\
\hline PHE27 & $\checkmark$ & $\checkmark$ & $\checkmark$ & $\checkmark$ & $\checkmark$ & $\checkmark$ & $\checkmark$ & $\checkmark$ & $\checkmark$ & $\checkmark$ & $\checkmark$ & $\checkmark$ \\
\hline TYR31 & $\checkmark$ & $\checkmark$ & $\checkmark$ & $\checkmark$ & $\checkmark$ & $\checkmark$ & $\checkmark$ & $\checkmark$ & $\checkmark$ & $\checkmark$ & $\checkmark$ & $\checkmark$ \\
\hline GLY115 & $\checkmark$ & $\checkmark$ & $\checkmark$ & $\checkmark$ & $\checkmark$ & $\checkmark$ & $\checkmark$ & $\checkmark$ & $\checkmark$ & $\mathbf{X}$ & $\checkmark$ & $\checkmark$ \\
\hline SER116 & $\checkmark$ & $\checkmark$ & $\checkmark$ & $\checkmark$ & $\checkmark$ & $\checkmark$ & $\checkmark$ & $\checkmark$ & $\checkmark$ & $\mathbf{X}$ & $\checkmark$ & $\checkmark$ \\
\hline ILE126 & $\checkmark$ & $\checkmark$ & $\checkmark$ & $\checkmark$ & $\checkmark$ & $\checkmark$ & $\checkmark$ & $\checkmark$ & $\mathbf{X}$ & $\checkmark$ & $\checkmark$ & $\checkmark$ \\
\hline TYR127 & $\checkmark$ & $\checkmark$ & $\checkmark$ & $\checkmark$ & $\checkmark$ & $\mathbf{X}$ & $\checkmark$ & $\checkmark$ & $\mathbf{X}$ & $\checkmark$ & $\checkmark$ & $\checkmark$ \\
\hline SER191 & $\checkmark$ & $\checkmark$ & $\checkmark$ & $\checkmark$ & $\checkmark$ & $\checkmark$ & $\checkmark$ & $\checkmark$ & $\checkmark$ & $\checkmark$ & $\checkmark$ & $\checkmark$ \\
\hline PHE238 & $\checkmark$ & $\checkmark$ & $\checkmark$ & $\checkmark$ & $\checkmark$ & $\checkmark$ & $\checkmark$ & $\checkmark$ & $\mathbf{X}$ & $\checkmark$ & $\checkmark$ & $\checkmark$ \\
\hline VAL239 & $\checkmark$ & $\checkmark$ & $\checkmark$ & $\checkmark$ & $\checkmark$ & $\checkmark$ & $\checkmark$ & $\checkmark$ & $\checkmark$ & $\checkmark$ & $\checkmark$ & $\checkmark$ \\
\hline ARG244 & $\checkmark$ & $\checkmark$ & $\checkmark$ & $\checkmark$ & $\checkmark$ & $\checkmark$ & $\checkmark$ & $\checkmark$ & $\checkmark$ & $\checkmark$ & $\checkmark$ & $\checkmark$ \\
\hline TYR247 & $\checkmark$ & $\checkmark$ & $\checkmark$ & $\checkmark$ & $\checkmark$ & $\checkmark$ & $\mathbf{X}$ & $\checkmark$ & $\checkmark$ & $\mathbf{X}$ & $\checkmark$ & $\mathbf{X}$ \\
\hline VAL319 & $\checkmark$ & $\checkmark$ & $\checkmark$ & $\checkmark$ & $\checkmark$ & $\checkmark$ & $\checkmark$ & $\checkmark$ & $\checkmark$ & $\checkmark$ & $\checkmark$ & $\checkmark$ \\
\hline TYR322 & $\checkmark$ & $\checkmark$ & $\checkmark$ & $\checkmark$ & $\checkmark$ & $\checkmark$ & $\checkmark$ & $\checkmark$ & $\checkmark$ & $\checkmark$ & $\checkmark$ & $\checkmark$ \\
\hline LEU323 & $\checkmark$ & $\checkmark$ & $\checkmark$ & $\checkmark$ & $\checkmark$ & $\checkmark$ & $\checkmark$ & $\checkmark$ & $\checkmark$ & $\checkmark$ & $\checkmark$ & $\checkmark$ \\
\hline
\end{tabular}

191 Conformation changes during the production runs were quantified using the root mean square

192 displacement (RMSD) on carbon alpha atom positions from structurally aligned frames for

193 DELLA and GID1A. The RMSD in the GID1A receptor for all simulations did not exceed $2.5 \AA$.

194 The RMSD of the disordered DELLA protein was found to have nearly double the RMSD of

195 GID1A, with maximums at $8 \AA$ and an average of approximately $4.5 \AA$. Breaking this down into

196 the MoRF (4) and inter-MoRF subunits, the DELLA containing MoRF 1 (residues 24 to 55), and

197 VHYNP containing MoRF 2 (residues 69 to 93) have similar levels of RMSD of $1.5 \AA \AA$. MoRF 3,

198 conserved YL(R/K)LIP residues, (residues 97 to 104) located at the C-terminus end of the GAI

199 N-terminus fragment modelled here has a slightly higher RMSD of $2 \AA$, which reflects having

200 fewer intra chain interactions compared to the other two MoRFs. As expected, the highest degree

201 of mobility is in the regions between the MoRFs - particularly at the $\mathrm{N}$ - and $\mathrm{C}$ - terminal regions

202 of the analysed fragment. The relatively lower RMSD found in the MoRFs compared to the 
203 linkers are also apparent in the apo form (aDG), meaning GA is removed from the binding

204 pocket.

205 As expected, the overall degree of mobility in the DELLA protein is reduced in the GA bound

206 forms compared to the apo form. Interestingly, the root mean square fluctuation (RMSF) for the

207 carbon alpha atoms in the region between MoRF 2 and MoRF 3 shows greater mobility with the

$208 \mathrm{GA}^{(\mathrm{a})}$ subset when compared with other GA variants analysed, shown in Figure 2 and Figure S1.

209 The GID1A residues were similarly analysed (Figure S9) and were found to be similar between

$210 \mathrm{aDG}$ and $\mathrm{GA}^{(\mathrm{a})} \cdot \mathrm{DG}$ systems. However, the gG systems exhibit an overall increase in RMSF

211 presumably due to the missing interactions with DELLA protein. The regions near pocket

212 residues of GID1A from Table 1 are found to be slightly less mobile in the gDG and gG systems

213 compared to the aDG system.

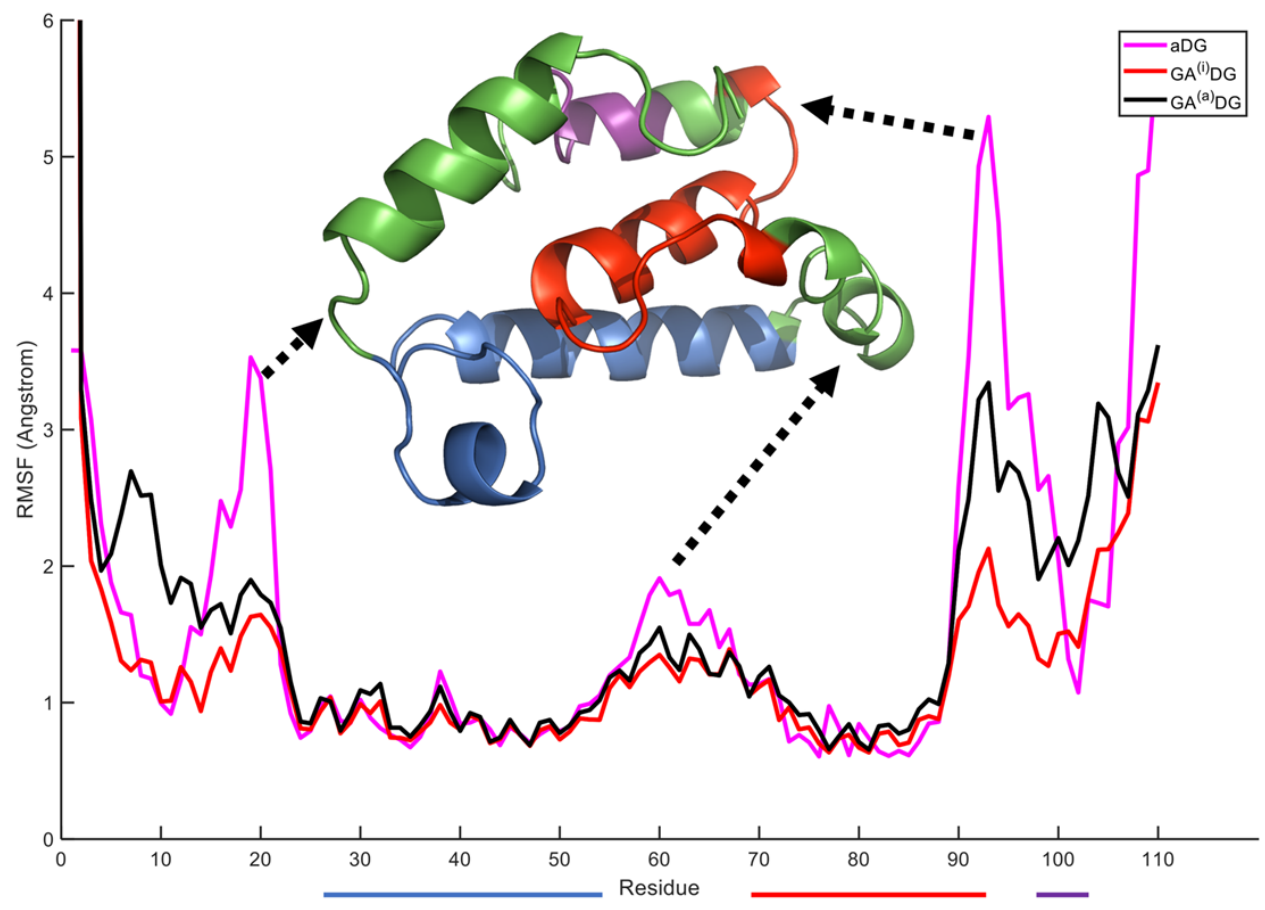


217 production runs per residue and averaging across all GA within the respective subgroups. The three coloured bars below the $x$ -

218 axis represent the MoRFs and match the colouring of these MoRFs on the GAl ribbon model of the N-terminus above. Dotted

219 arrows match key flexible parts of the model with the mean RMSF values.

220 GAx tRAMD analysis: Channel and cleft pathways

221 After $20 n s$ production runs (computational simulations) were completed, this information was

222 used in tRAMD to identify the $\mathrm{GA}^{(\mathrm{x})}$ egression pathways during the dissociation process. We

223 observed that there are essentially two egression pathways, as shown in Figure 3. The longer

224 pathway uncovers the previously reported channel pathway by Hao et al. (28). The shorter

225 pathway is dubbed cleft because the opening is readily closed by the presence of DELLA motif

226 and the egressions yielded from this route have fewer interactions overall than in the channel

227 pathway in the GID1A systems.
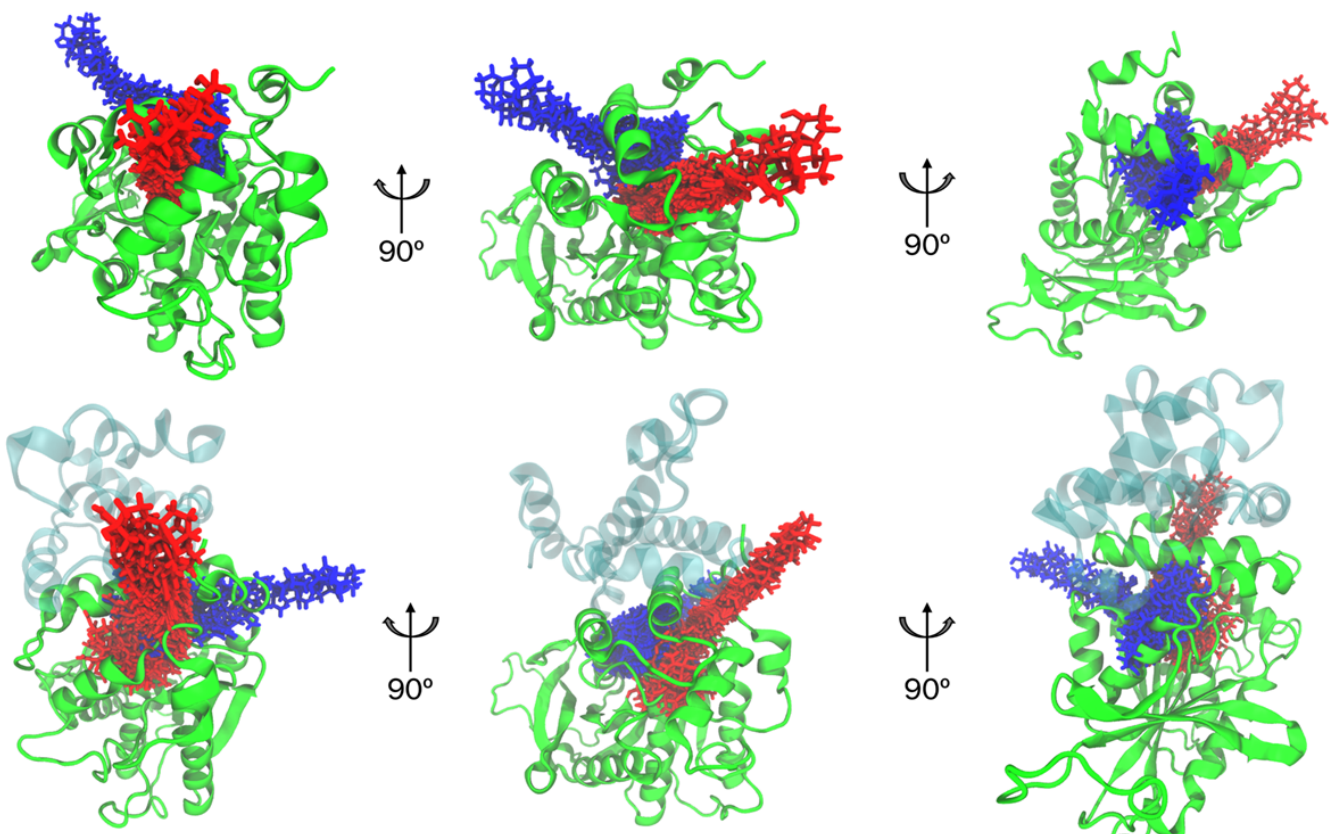

Figure 3: Representations of pathways in GID1A system. Bottom DELLA in cyan and GID1A in green, top GID1A. The blue

230 pathway is a newly discovered GID1 cleft, red is known channel pathway. Middle perspective looks down alpha helix B of GID1A

231 with N-terminal to the right. 
232 It is worth noting that Hao et al. used an incomplete gDG structural model, taken directly from

233 the crystal structure (PDB accession code: 2ZSH). Nevertheless, the channel can be identified

234 within that static structure. Subsequently, Hao et al. performed free energy calculations using

235 umbrella sampling for $\mathrm{GA}_{4}$ as it moved into the binding pocket along this identified pathway.

236 Working inward, from the outside surface of GID1A toward the internal binding pocket, Hao et.

237 al. determined the initial residues that interact with $\mathrm{GA}_{4}$ along this pathway (that we now call the

238 channel) to be ASP243 and THR240 (note we have not retained all the same residue numbering

239 as in Hao et al., see supplementary file for explanation). Then a rotation of $\mathrm{GA}_{4}$ occurs to

240 interact with TYR247 and ASP243 to allow chaperoning to more buried interactions with

241 HIS119 and ARG244. Finally, GA 4 resides in the binding pocket with its carboxyl group

242 interacting strongly with SER116 and SER191. Excluding the two binding pocket serine

243 interactions in the docked state, these six residues were identified by Hao et al. and are used here

244 to identify the same pathway in our data using a large set of $\mathrm{GA}^{(\mathrm{x})}$.

245 For each GA variant, the set of GID1A residues that it interacts with when leaving through the

246 channel are summarized in Table 2. In most systems the previously identified hydrogen bonding

247 residues from Hao et al. are recurrent. It is notable that most of the hydrophobic contact residues

248 are simply adjacent to these residues, but are highly conserved across the phylogeny of GID1.

249 The only recurrent residue with large discrepancy between the $\mathrm{GA}^{(\mathrm{a})}$ and $\mathrm{GA}^{(\mathrm{i})}$ subsets is ASN32.

250 Unlike the pocket residue interactions, the $\mathrm{GA}^{(\mathrm{i})}$ and $\mathrm{GA}^{(\mathrm{a})}$ interacted similarly with channel

251 residues along the egressions. In simulations without the GAI N-terminus (gG systems), GID1A

252 was found to be flexible enough to allow egression from this same pathway without a large

253 portion of the interactions reported in Hao et al., Table S1. Residue interactions we identified in

254 the channel pathway in both the gG and the gDG systems were THR240, ASN32, and MET220. 
Table 2: Hydrogen bonding and contact recurrent residues during Channel pathway egressions gDG (* denotes reported in Hao

et al. as important pathway residues). Recurrent hydrogen bonds, in blue, and hydrophobic contact residues, in black, during

263 production runs of the gDG system. GA variants in bold are reported bioactive. Recurrent set generated from GA(a) subset and

264 any pocket residue reported in Table 1 is removed for clarity, and otherwise, $\boldsymbol{X}$ denotes absence of interaction in given system,

265 while check denote presence of interaction.

\begin{tabular}{|c|c|c|c|c|c|c|c|c|c|c|c|c|}
\hline $\operatorname{Res}^{\mathrm{GA}}$ & 1 & 3 & 4 & 7 & $12^{160 x}$ & 20 & 9 & 8 & 12 & $4^{\mathrm{MeO}}$ & $4^{16 / 17 o x}$ & 34 \\
\hline LYS28 & $\checkmark$ & $\checkmark$ & $\checkmark$ & $\checkmark$ & $\checkmark$ & $\checkmark$ & $\checkmark$ & $\checkmark$ & $\checkmark$ & $\checkmark$ & $\checkmark$ & $\checkmark$ \\
\hline ASN32 & $\checkmark$ & $\checkmark$ & $\sqrt{ }$ & $\sqrt{ }$ & $\checkmark$ & $x$ & $x$ & $x$ & $\checkmark$ & $x$ & $x$ & $x$ \\
\hline LEU34 & $\checkmark$ & $\checkmark$ & $\sqrt{ }$ & $\sqrt{ }$ & $\checkmark$ & $\checkmark$ & $\sqrt{ }$ & $\checkmark$ & $\checkmark$ & $x$ & $\sqrt{ }$ & $\checkmark$ \\
\hline PHE41 & $\checkmark$ & $\checkmark$ & $\checkmark$ & $\sqrt{ }$ & $\checkmark$ & $\checkmark$ & $\checkmark$ & $\checkmark$ & $\checkmark$ & $x$ & $\checkmark$ & $\checkmark$ \\
\hline HIS119* & $\checkmark$ & $\checkmark$ & $\checkmark$ & $\sqrt{ }$ & $\checkmark$ & $\checkmark$ & $\checkmark$ & $\checkmark$ & $\checkmark$ & $\checkmark$ & $\checkmark$ & $\checkmark$ \\
\hline ASP190 & $\checkmark$ & $\checkmark$ & $\checkmark$ & $\sqrt{ }$ & $\checkmark$ & $\checkmark$ & $\checkmark$ & $\checkmark$ & $\checkmark$ & $\checkmark$ & $\checkmark$ & $\checkmark$ \\
\hline MET220 & $\checkmark$ & $\checkmark$ & $\checkmark$ & $\sqrt{ }$ & $\checkmark$ & $\checkmark$ & $\checkmark$ & $\checkmark$ & $\checkmark$ & $\checkmark$ & $\checkmark$ & $\checkmark$ \\
\hline THR240* & $\checkmark$ & $\checkmark$ & $\checkmark$ & $\sqrt{ }$ & $\checkmark$ & $\checkmark$ & $\checkmark$ & $\checkmark$ & $\checkmark$ & $\checkmark$ & $\checkmark$ & $\checkmark$ \\
\hline ASP243* & $\checkmark$ & $\checkmark$ & $\checkmark$ & $\checkmark$ & $\checkmark$ & $\checkmark$ & $\checkmark$ & $\checkmark$ & $\checkmark$ & $x$ & $\checkmark$ & $\checkmark$ \\
\hline TRP246 & $\checkmark$ & $\checkmark$ & $\checkmark$ & $\checkmark$ & $\checkmark$ & $\checkmark$ & $\checkmark$ & $\checkmark$ & $\checkmark$ & $\checkmark$ & $\checkmark$ & $\checkmark$ \\
\hline TYR247* & $\checkmark$ & $\checkmark$ & $\checkmark$ & $\checkmark$ & $\checkmark$ & $\checkmark$ & $\checkmark$ & $\checkmark$ & $\checkmark$ & $\checkmark$ & $\checkmark$ & $\checkmark$ \\
\hline LEU290 & $\checkmark$ & $\checkmark$ & $\checkmark$ & $\sqrt{ }$ & $\checkmark$ & $\checkmark$ & $\checkmark$ & $\checkmark$ & $\checkmark$ & $\sqrt{ }$ & $\sqrt{ }$ & $\checkmark$ \\
\hline
\end{tabular}

267 A second pathway is also uncovered, as shown in Figure 3 in blue. Without the DELLA protein,

268 this pathway is slightly shorter than the channel even with dynamic conformations considered, 
ca. 6-8 $\AA$ vs. 9-12 $\AA$. This cleft pathway is also more flexible than the channel with a cross-

270 section of $8 \AA$ that can expand up to $14 \AA$, versus the channel with 9 to $11 \AA$ during egressions.

271 Noting the differences in the blue egression plots between the top and bottom panels in Figure 3,

272 the addition of the DELLA subunit causes a major change to the cleft egression path. While the

273 addition does not heavily perturb the channel pathway, the cleft pathway must egress closer to

274 the N-terminal of GID1A near MoRF 1 of GAI. The cleft pathway is visualized in Figure 4 and

275 Figure S2. The channel pathway requires rotation around the shorter side of the GA molecule,

276 while this cleft requires only minor rotation to align the molecule. Assistance with this alignment

277 is offered from the electropositive interior of the pocket by attracting the carboxylate moiety and

278 position the GA to bind into the pocket. Both the matching shape and electrostatics show a clear

279 binding pathway, which is a persistent signature in all the simulations. Movie S1 and S2

280 provides a dynamic comparison of the egression pathways and their essential dynamics through

281 the channel and cleft pathways, with and without the DELLA protein.
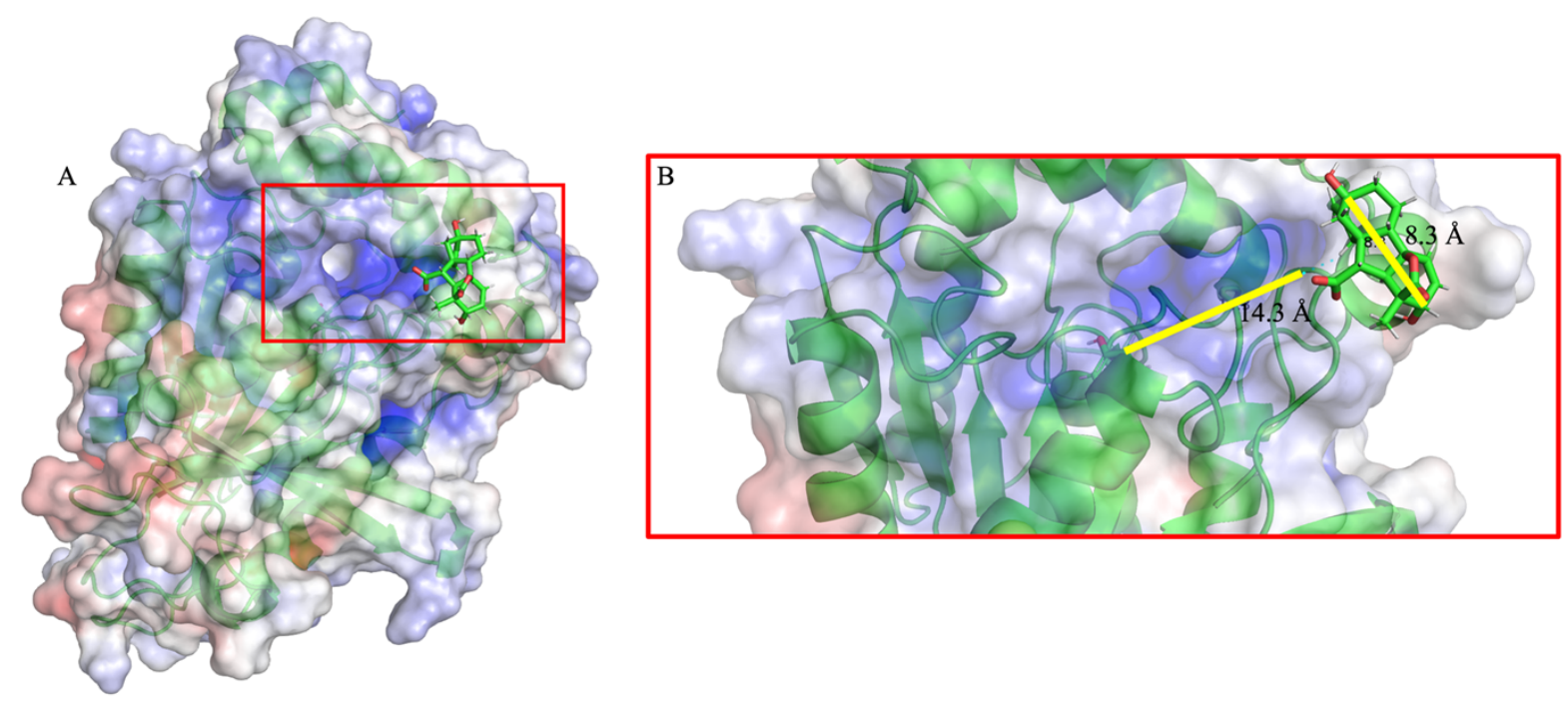
285 When the DELLA protein is bound to GID1A, the cleft opening is blocked. Nevertheless, as

286 Figure 3 indicates, $\mathrm{GA}^{(\mathrm{x})}$ can egress from the cleft pocket opening even in the presence of the

287 DELLA protein, albeit in a heavily perturbed pathway. The binding pocket residence times for

288 the set of GA variants considered here for the gDG system are summarized in Table 3. On

289 average the egression pathways through the channel is of higher propensity than the cleft

290 pathway. However, among the $12 \mathrm{GA}$ variants considered here, $\mathrm{GA}_{9}, \mathrm{GA}_{4}$ and $\mathrm{GA}_{34}$ are

291 exceptions that exhibit a higher propensity to leave the binding pocket through the cleft pathway.

292 The dipole moment calculated for each molecule displays little correlation to average residence

293 time, as many of the $\mathrm{GA}^{(\mathrm{a})}$ share very similar values.

Table 3: Residence times of GA in gDG and observed pathways from tRAMD egression experiments. Known $G A^{(a)}$ data are

\begin{tabular}{|l|c|c|c|c|c|}
\hline System GA & Dipole (Debye) & Channel Obs. & Cleft Obs. & Avg residence (ns) & $\boldsymbol{\sigma}$ \\
\hline $\mathbf{3}$ & 15.93 & 8 & 2 & 0.91 & 0.09 \\
\hline $\mathbf{4}$ & 15.05 & 5 & 7 & 0.87 & 0.15 \\
\hline $\mathbf{1}$ & 15.97 & 6 & 4 & 0.79 & 0.12 \\
\hline $\mathbf{7}$ & 15.46 & 7 & 3 & 0.28 & 0.09 \\
\hline $\mathbf{1 2 - 1 6 0 x}$ & 18.97 & 7 & 3 & 2.90 & 0.37 \\
\hline $\mathbf{9}$ & 13.07 & 3 & 7 & 0.39 & 0.07 \\
\hline 20 & 13.94 & 10 & 1 & 0.84 & 0.16 \\
\hline 8 & 14.75 & 8 & 2 & 0.37 & 0.10 \\
\hline 12 & 18.06 & 5 & 5 & 2.30 & 0.40 \\
\hline $4 \mathrm{MeO}$ & 5.92 & 5 & 5 & 0.09 & 0.03 \\
\hline 34 & 11.37 & 4 & 6 & 0.70 & 0.24 \\
\hline $4-16 / 170 x$ & 10.20 & 8 & 2 & 1.23 & 0.32 \\
\hline
\end{tabular}

297 Given that the GAI MoRF 1 blocks the cleft, the tRAMD method was applied to the gG system

298 for a subset of $\mathrm{GA}^{(\mathrm{x})}$ when the cleft is open. It was found that the $\mathrm{GA}^{(\mathrm{x})}$ have a choice of

299 egressing out of the binding pocket through the cleft or the channel. The residence times found

300 for the subset of $\mathrm{GA}^{(\mathrm{x})}$ with respect to the $\mathrm{gG}$ system are summarized in Table 4 . Across the GA 
301 variants considered, on average, there is greater propensity to egress out of the cleft. However,

$302 \mathrm{GA}_{20}$ and $\mathrm{GA}_{12-160 x}$ represent two exceptions. Therefore, it is clear that both egression pathways

303 are viable in both the $\mathrm{gDG}$ and $\mathrm{gG}$ complexes for all $\mathrm{GA}^{(\mathrm{x})}$, with a variable degree of propensity

304 for the cleft over the channel.

Table 4: Residence times of GA in $g G$ and observed pathways from tRAMD egression experiments. Known $G A^{(a)}$ data are shaded

grey. Average residence time of total egressions for given $G A^{(x)} g G$ system, with the standard deviation also reported.

\begin{tabular}{|c|c|c|c|c|c|}
\hline System & Dipole (Debye) & Channel Obs. & Cleft Obs. & Avg residence (ns) & $\boldsymbol{\sigma}$ \\
\hline $\mathbf{3}$ & 15.93 & 2 & 8 & 0.28 & 0.04 \\
\hline $\mathbf{4}$ & 15.05 & 3 & 7 & 0.25 & 0.05 \\
\hline $\mathbf{7}$ & 15.46 & 3 & 7 & 0.08 & 0.02 \\
\hline $\mathbf{1 2 - 1 6 0 x}$ & 18.97 & 5 & 5 & 1.03 & 0.21 \\
\hline 20 & 13.94 & 6 & 4 & 0.18 & 0.05 \\
\hline 12 & 18.06 & 3 & 7 & 0.47 & 0.10 \\
\hline $4 \mathrm{MeO}$ & 5.92 & 2 & 8 & 0.04 & 0.005 \\
\hline
\end{tabular}

310 These data show that residence time alone does not offer a simple explanation for bioactivity.

311 Even within the known bioactive set of GA ligands there is variation in average residence times

312 particularly between $\mathrm{GA}_{7}$ and $\mathrm{GA}_{12-160 x}$. To glean a mechanistic explanation, each egression

313 pathway was analysed separately where the counting of hydrogen bonds and hydrophobic

314 contacts during production runs found that $\mathrm{GA}_{7}$ had significantly more hydrogen bonding to

315 residues TYR247, GLY115, and VAL319. During these progression runs the GA 7 ligand

316 exhibited greater mobility than any of the other putative active $\mathrm{GA}^{(\mathrm{x})}$ ligands, in both the gG and

317 gDG systems. A simple mechanistic explanation for markedly short egression times in $\mathrm{GA}_{7}$

318 could be differences in mobility within the pocket of the DELLA - GID1A complex due to the 
bioRxiv preprint doi: https://doi.org/10.1101/2020.12.15.422840; this version posted December 15,2020 . The copyright holder for this preprint (which was not certified by peer review) is the author/funder, who has granted bioRxiv a license to display the preprint in perpetuity. It is made available under aCC-BY 4.0 International license.

319 lack of an alcohol group at C13. However, no correlation was found between RMSD values and 320 egression times across the $\mathrm{GA}^{(\mathrm{x})}$ to support such an explanation, Figure S5. 
Table 5: Hydrogen bonding and contact recurrent residues during Cleft pathway egressions gG. Recurrent hydrogen bonds, in

323 blue, and hydrophobic contact residues, in black, during production runs of the gDG system. Recurrent set generated from GA(a)

324 subset and any pocket residue reported in Table 1 is removed for clarity, and otherwise, $x$ denotes absence of interaction in

325 given system, while checks denote presence of interaction.

\begin{tabular}{|l|l|l|l|l|l|l|l|}
\hline \multicolumn{1}{|c|}{ GA } & 3 & $\mathbf{4}$ & $\mathbf{7}$ & $\mathbf{1 2}^{\mathbf{1 6 0 x}}$ & $\mathbf{2 0}$ & 12 & $4^{\mathrm{MeO}}$ \\
\hline LEU23 & $\checkmark$ & $\checkmark$ & $\checkmark$ & $\checkmark$ & $\checkmark$ & $\checkmark$ & $\checkmark$ \\
\hline LYS28 & $\checkmark$ & $\checkmark$ & $\checkmark$ & $\checkmark$ & $\checkmark$ & $\checkmark$ & $\mathbf{X}$ \\
\hline LEU49 & $\checkmark$ & $\checkmark$ & $\checkmark$ & $\checkmark$ & $\mathbf{X}$ & $\checkmark$ & $\checkmark$ \\
\hline ARG51 & $\checkmark$ & $\checkmark$ & $\checkmark$ & $\checkmark$ & $\mathbf{X}$ & $\checkmark$ & $\checkmark$ \\
\hline HIS119 & $\checkmark$ & $\checkmark$ & $\checkmark$ & $\checkmark$ & $\mathbf{X}$ & $\checkmark$ & $\checkmark$ \\
\hline SER121 & $\checkmark$ & $\checkmark$ & $\checkmark$ & $\checkmark$ & $\checkmark$ & $\checkmark$ & $\checkmark$ \\
\hline MET220 & $\checkmark$ & $\checkmark$ & $\checkmark$ & $\checkmark$ & $\mathbf{X}$ & $\checkmark$ & $\checkmark$ \\
\hline LEU290 & $\checkmark$ & $\checkmark$ & $\checkmark$ & $\checkmark$ & $\mathbf{X}$ & $\checkmark$ & $\checkmark$ \\
\hline ILE291 & $\checkmark$ & $\checkmark$ & $\checkmark$ & $\checkmark$ & $\mathbf{X}$ & $\checkmark$ & $\checkmark$ \\
\hline GLY320 & $\checkmark$ & $\checkmark$ & $\checkmark$ & $\checkmark$ & $\checkmark$ & $\checkmark$ & $\checkmark$ \\
\hline
\end{tabular}

327 Analysis of the contact residues in the cleft pathway are shown in Table 5. In the complexed

328 gDG system the cleft pathway is more complicated as the GAI MoRF 1 largely covers the cleft,

329 but the space for this pathway is intrinsically present in the binding pocket of GID1A. As a GA ${ }^{(\mathrm{x})}$

330 leaves GID1A in the gDG through the cleft, it must directly pass the DELLA residues in MoRF 1

331 near GAI-LEU32, as highlighted in Table S2, and the nearby GID1A residue LEU217.

333 As a complementary analysis to removing the GA ligand from the complex pocket, tRAMD

334 egressions on the GAI N-terminus were also performed to explore the coordination of DELLA

335 onto GID1A. This was performed for all gDG systems previously discussed including the apo

336 DELLA - GID1A system (aDG). Important residue subsets described as MoRFs are known to be

337 conserved features of the GAI N-terminus and considered to be responsible for recognizing and 
338 binding to GID1A [22]. The residues for MoRF 1 and 2 were included in the Hao modelling but

339 the residues linking MoRFs 1 and 2 and beyond were missing from their model. Nevertheless,

340 Hao et al. concluded there are differences between holo, with $\mathrm{GA}_{4}$, and the apo DELLA · GID1A

341 complex at the incomplete protein interface. DELLA protein residues, most of which are within

342 or near the later part of MoRF 1, were found in that study to change interaction by conformation

343 changes induced when $\mathrm{GA}_{4}$ was present in GID1A. These residues interacted with GID1A N-

344 terminal residues LEU9 and ASN32 on the alpha helix lid of GID1A. Although it was concluded

345 that $\mathrm{GA}_{4}$ recognition leads to a conformationally stabilizing response, the previous work was

346 mainly informed by the static protein structure from X-ray crystallography. In light of these

347 critical differences we analysed both residence time (Table 6) and key contact residues for the

348 MoRFs in tRAMD uncapping modelling experiments.

349 Table 6: Residence times of GAI N-terminus uncapping from various GID1A systems, with the standard deviation reported.

350 Known $G^{(a)}$ data are shaded grey.

\begin{tabular}{|l|c|c|c|l|}
\hline System GA & $\begin{array}{l}\text { Dipole } \\
\text { (Debye) }\end{array}$ & MD eq. (ns) & \multicolumn{1}{l|}{$\begin{array}{l}\text { Avg } \\
\text { residence } \\
\text { (ns) }\end{array}$} & \\
\hline APO & 15.93 & 20 & 0.75 & 0.15 \\
\hline $\mathbf{3}$ & 15.93 & 60 & 0.92 & 0.21 \\
\hline $\mathbf{4}$ & 15.05 & 20 & 1.90 & 0.53 \\
\hline $\mathbf{1}$ & 15.97 & 20 & 2.56 & 0.38 \\
\hline $\mathbf{7}$ & 15.46 & 20 & 2.34 & 0.79 \\
\hline $\mathbf{1 2 - 1 6 0 x}$ & 18.97 & 20 & 2.88 & 0.48 \\
\hline $\mathbf{9}$ & 13.07 & 20 & 1.42 & 0.44 \\
\hline 20 & 13.94 & 20 & 0.75 & 0.16 \\
\hline $\mathbf{8}$ & 14.75 & 20 & 0.58 & 0.11 \\
\hline 12 & 18.06 & 20 & 2.84 & 0.63 \\
\hline $4 \mathrm{MeO}$ & 5.92 & 20 & 3.60 & 0.72 \\
\hline 34 & 11.37 & 20 & 0.64 & 0.15 \\
\hline $4-16 / 170 x$ & 10.20 & 20 & 1.01 & 0.29 \\
\hline
\end{tabular}


352 The hydrogen bond and hydrophobic contact analysis was performed for the DELLA egressions

353 as done for GA ligands. Cursory viewing of the full DELLA · GID1A complex shows MoRF 1

354 and 2 are largely responsible for stable binding to GID1A. MoRF 3 is above the entire complex,

355 but note that this model is lacking a large flexible linker region between the $\mathrm{N}$-terminus and

356 GRAS domain, as well as the remaining GRAS domain. We acknowledge that it is possible that

357 MoRF 3 could interact differently than observed in our simulations when the entire GRAS

358 domain is modelled. With this caveat, we analyse MoRF 3 similarly to the other two MoRFs.

359 The analysis of all three MoRF contacts during uncapping shows that the DELLA subunit has

360 long-lasting hydrogen bonds to GID1 in gDG. As an exception, only MoRF 3 maintained its

361 local secondary structure, and it loses intra chain interactions most rapidly. Since there is a long

362 flexible linker between the GRAS domain and MoRF 3, the weaker interaction with MoRF 3 is

363 expected.

364 The residues previously identified as important in GID1A LEU9 and ASN32 were found to be

365 entirely conserved when analysing the hydrogen bond and contact interactions with MoRF 1

366 during production runs of the complex containing the $\mathrm{GA}^{(\mathrm{a})}$ ligands $1,3,4,7$, and 12-16ox.

367 Hydrogen bonding to GID1A residues 9 and 32 were not statistically different between GA ${ }^{(a)}$.

368 DG and the apo aDG systems. Comparing the interaction behaviour of the residues of MoRF 1

369 between $\mathrm{GA}^{(\mathrm{a})}$ and $\mathrm{GA}^{(\mathrm{i})}$, only LEU9 was found to have conserved contacts.

370 Analysing the uncapping of DELLA (Table 6), shows a weak correlation with the bioactive GA

371 systems taking longer to remove the DELLA subunit. An average of $2.12 \mathrm{~ns}$ for the GA ${ }^{(a)}$

372 systems versus $1.55 \mathrm{~ns}$ for the $\mathrm{GA}^{(\mathrm{i})}$ variants, with APO having an average of 0.75 . Notably

$373 \mathrm{GA}_{4 \mathrm{MeO}}$ and $\mathrm{GA}_{12}$ significantly increase the average residence time of the DELLA protein for the

$374 \mathrm{GA}^{(\mathrm{i})}$ category. During uncapping events the ligand pocket seemed relatively undisturbed, e.g. 
375 the ligand did not lose or gain many new interactions in all systems. For all GA ligands, the

376 series of key pocket GA contact residues mentioned in Table 1 were recurrent in these uncapping

377 simulations as well.

378 MoRF 1 also showed long lasting hydrogen bonds to ASN19, LYS28, ARG51, and ASN326

379 residues of GID1A. Interaction with the N-terminal of GID1A were found to be largely

380 stabilized by hydrophobic interactions. While both of these interaction sets persist for a large

381 number of the uncapping events, the interactions often break before the end of the DELLA

382 egression. It is notable that the DELLA-GLU26 from the eponymous DELLA motif of MoRF 1

383 directly interacts with ASN19 of GID1A and was observed to be long-lasting during uncapping.

384 Analysis of MoRF 2 shows recurrent interactions of the active GA systems are largely intra

385 chain interactions, suggesting MoRF 2 controls the tertiary structure of the DELLA subunit.

386 MoRF 2 also maintains long-lasting hydrogen bonds to TYR48 and ARG51 of GID1A.

387 Comparisons between the $\mathrm{GA}^{(\mathrm{x})}$ systems and the APO system hydrogen bonding of MoRF 2

388 showed inter chain residue interactions to GID1A residue ARG51 to interact significantly more

389 in the APO system. GID1A's ARG51 likely swings between MoRF 1 and 2 during normal

390 dynamics.

391 MoRF 3 was expected to only interact with intra-chain residues within DELLA due to its

392 position above the entire complex (Figures 1 and 5). Interactions of the main bioactive GA

393 systems showed the expected intra-chain interactions to consist of highly localized residues,

394 within plus or minus five residues of MoRF 3. However, between the gDG and aDG systems the

395 APO system exhibited unique interactions with, MET13, ALA41, and ASP42 of the GAI N-

396 terminus. These are significantly farther away than any interaction in the bioactive systems, 
398 a GA ligand, as visualized in Figure 5.

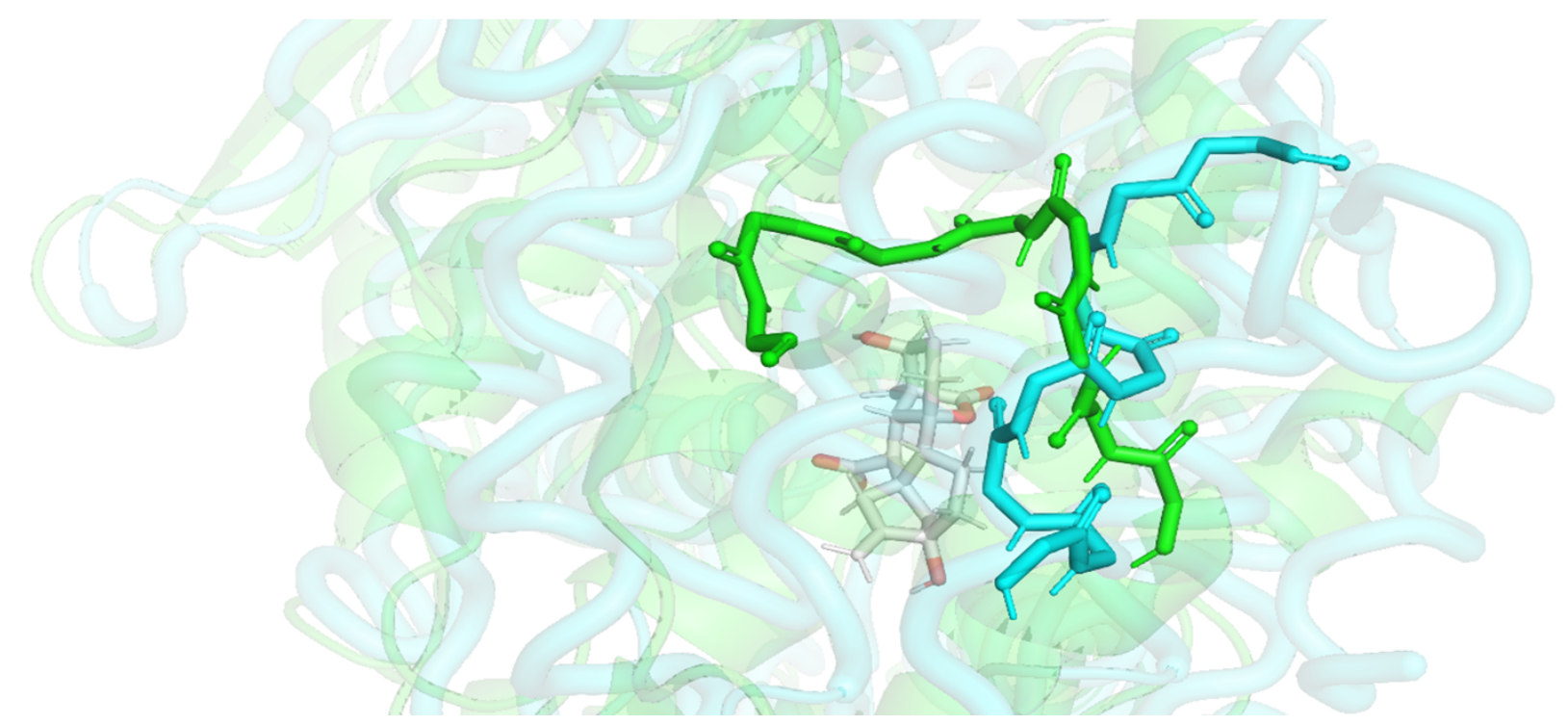

Figure 5: MoRF 3 conformations of typical active gDG, cyan, system compared with apo (aDG), green.

402 To examine the phylogeny of GID1 across a broad cross-section of plants from divergent taxa, 403 we mined the existing database EnsemblPlants (34). Upon examining the phylogenetic tree for

404 this family, it is clearly split into four major subtrees. These four clades consist of 1) basal 405 Angiosperms and Moss ancestral plant species, 2) monocot GID1 (one major branch only with

406 duplications in later parts of the phylogeny), and two major dicot branches 3) the GID1A and 4)

407 GID1B branches - the latter two housing the Arabidopsis GID1A and GID1B members

408 respectively. Comparing these trees with the plant evolutionary tree of life suggests several

409 duplication events have occurred in the GID1A and GID1B branches (identified as duplication or

410 ambiguous nodes in the tree). These factors were combined to derive 26 major subgroupings

411 from which consensus sequences were created by setting conservation at $90 \%$. This cut-off of

$41210 \%$ protein variation for proteins spanning the $50-100$ million years in evolution of these 
413 suborders and superclasses is equivalent to 5-50 times lower per million years (/m.y.) when

414 compared to the degree of conservation between alleles $(0.1-0.2 \% / \mathrm{m} . \mathrm{y}$. versus $2-3 \%$ allelic

415 variation separated by short time periods of the order of a few million years at most and equating

416 to approximately $1-5 \% / \mathrm{m} . \mathrm{y}$.$) . We suggest this cut-off is a conservative measure and identifier$

417 of key conserved residues across broad taxonomic groups of plant families.

418 We examined the conservation at 37 key $\mathrm{GA}^{(\mathrm{x})}$ contact residues identified in the computational

419 analysis across the four main GID1 groups (basal GID1, monocot GID1, dicot GID1A and dicot

420 GID1B, results detailed in Supplementary Tables 3-5). In summary 11 out of the 15 pocket

421 contact residues show a high degree of conservation and the remaining 4 only show conservative

422 substitutions, 10 out of 12 channel contact residues showed a high degree of conservation (some

423 with a degree of conservative substitutions) and only 2 showed non-conservative substitutions

424 while 8 out of 10 cleft residues show a high degree of conservation and the remaining 2 only

425 show conservative substitutions. These results suggest a high degree of conservation across all of

426 the GID1 residues that contact the GA ligand. The key GID1 cap residues (alpha helix A, B and

427 C) in contact with the MoRFs of DELLAs are also highly conserved or have conservative

428 substitutions (data not shown).

429 Discussion

430 The influence of cleft, channel and MoRFs on GA binding

431 The description for how DELLA GAI binds to GID1A has common aspects across all our

432 models. First, the GID1A lid must be closed for DELLA GAI to bind to GID1A. In absence of

433 direct experimental evidence, it is presumed that the DELLA GAI protein only binds to GID1A

434 in the presence of GA. Once formed, the gDG complex subsequently attracts an E3 ubiquitin 
435 ligase (SLEEPY1 in Arabidopsis), a component of the ubiquitin-mediated machinery that

436 initiates a programmed degradation of the attached DELLA protein. All prior work neglected to

437 consider the effect of GA variants by assuming that only bioactive GA plays a role in the earliest

438 part of this process. Here, we also considered the possible effects of inactive GA on the DELLA

439 binding process, which is important for developing a complete kinetic model.

440 We found that the same application of force in tRAMD simulations produce egressions of GA(x)

441 from the gG and gDG systems. Not-withstanding exceptions, a general trend indicates there is

442 higher propensity for cleft pathway egressions in gG and channel pathway egressions in gDG.

443 Furthermore, any GA ligand present in the DELLA · GID1 complex is able to stabilize the GAI

444 subunit in its attachment to GID1A. This is suggested by results from comparing uncapping

445 residence times, finding that they are, on average, higher for all tested gDG systems than for the

446 aDG systems. This result applies to each $\mathrm{GA}^{(\mathrm{x})}$ case studied separately. Taking this result alone,

447 we cannot discriminate any critical differences between $\mathrm{GA}^{(\mathrm{x})}$ that would classify them as

448 bioactive or inactive. This is not to say there are no differences. Consistent with our production

449 run results, it is likely that the different $\mathrm{GA}^{(\mathrm{x})}$ create differences in conformational dynamics

450 within the gDG complexes (not studied here) that affect the degradation of the attached DELLA

451 protein.

452 Relatively lower RMSD was found in the MoRFs compared to the linkers between the MoRFs in

453 both gDG and aDG systems. Overall mobility in the DELLA protein is less in gDG compared to

$454 \mathrm{aDG}$ for each $\mathrm{GA}^{(\mathrm{x})}$. As Figure 2 shows, the region between MoRF 2 and MoRF 3 has greater

455 mobility with known bioactive GA compared to known inactive GA variants. The RMSF for the

456 carbon alpha atoms in the key interacting pocket residues of GID1A are found to be less mobile

457 in the aDG and $\mathrm{gG}$ systems compared the gDG systems. In contrast, the DELLA protein RMSF 
458 has greater mobility in aDG than found within the gDG systems, see Figure 2 and Figures S8 and

459 S9 for additional RMSF data. These comparisons were made across our $\mathrm{GA}^{(\mathrm{x})}$ set. Shifts in

460 mobility from one region to another is easily understood by an enthalpy/entropy compensation

461 mechanism driven by rigidity changes (2). Conformational entropy increases in certain regions

462 that become more flexible, while other regions become more rigid with more favourable

463 enthalpy due to conformational shifts in a protein. The greater RMSF found in the DELLA

464 protein when complexed in the aDG system is consistent with experimental data that without GA

465 the DELLA protein does not bind to GID1A. In essence, any of the $\mathrm{GA}^{(\mathrm{x})}$ forms can act as a

466 cofactor. Furthermore, if GA leaves gDG, it is expected that aDG becomes an unstable structure

467 with a relatively short lifetime relative to the gDG lifetime. However, our data show it has a

468 relatively long lifetime relative to GA binding to aDG through the channel pathway. These

469 examples show that the DELLA protein modifies the inherent dynamics of GID1, with details

470 that depend on the GA variant bound to GID1 and whether it is in apo form.

\section{Sequence of events and bioactivity of Gibberellin}

472 Determining the bioactivity for GA variants is an ongoing challenge of exploration, as illustrated

473 by the recent addition of DHGA12 into the bioactive category (33). With this line of

474 classification, there is an underlying assumption that appropriate concentrations of specific GA

475 variants must be present at certain plant tissues or cells. An intricate web of GA modifying

476 enzymes exists and these are clearly differentially regulated under particular environmental

477 stimuli (35). These modification systems presumably shift GA ${ }^{(\mathrm{x})}$ population abundance at

478 particular cellular locations. However, a mixture of GA variants will likely be present at most

479 locations. Unless there is an overwhelmingly strong differentiation between bioactive and

480 inactive GA, the binding process that takes place to form gDG should account for competitive 
481 binding. Our results show that residence times of GA variants do not correlate to bioactivity.

482 There is a trend in our data that residence time increases when the GA moieties support more

483 intra-molecular H-bonding. These results suggest there are subtle mechanisms in play that

484 govern the observed specificity in plant growth regulation. To understand these differences

485 requires looking at a specific GA moiety along with propensities for the GA variant to form $\mathrm{H}$ -

486 bonding and hydrophobic contacts within the cleft and channel pathways, which will affect

487 association and dissociation rates.

488 In the absence of GAI, entry for any $\mathrm{GA}^{(\mathrm{x})}$ via the cleft pathway is observably more accessible

489 than via the channel pathway. This is demonstrated by the fewer hydrogen bond interactions in

490 cleft egressions in Table 5 when compared to channel egressions in Table S1 in the GID1A

491 system. Along with the easier arrangement of GA carboxylate to organize into the pocket and the

492 higher likelihood of observation of cleft egression (see Table 4), this suggests an easier route in

493 the absence of DELLA motif. However, when GAI is bound to GID1A, by MoRF 1 and 2, the

494 contact residues to GA at the surface of the cleft pathway and the side chain flexibility needed to

495 open the cleft pathway are hindered. Egressions along the cleft pathway were still observed with

496 GAI present but drastically altered. In the complexed state GA was forced to take paths directly

497 under the DELLA MoRF 1 segment (see GAI-LEU32 contacts in Table S2). This suggests that

$498 \mathrm{GA}^{(\mathrm{x})}$ egressions from the closed cleft will destabilize the gDG structure, as hydrophobic

499 interactions at this interface are disturbed. This in turn could help control the necessary GA ${ }^{(a)}$

500 recognition, as the more bioactive GA ligands are less likely to leave or to interact with this

501 aliphatic region. MoRF 2 interactions are found to be similar between the $\mathrm{GA}^{(\mathrm{a})}$ and $\mathrm{GA}^{(\mathrm{i})}$

502 systems, removing it as a possible source for discrimination. We also note that a number of the

503 channel contacts evident in $\mathrm{GA}^{(\mathrm{x})}$ egression pathways are missing when the ligand is GA ${ }^{(\mathrm{i})}$. Thus, 
504 there is a higher propensity for $\mathrm{GA}^{(\mathrm{i})}$ to be expunged from the closed cleft than a $\mathrm{GA}^{(\mathrm{a})}$.

505 Dynamical differences in the intra-chain interactions within DELLA are observed when

506 bioactive gDG and aDG are compared.

507 The opening of the cleft pathway in GID1 is a hole that can fit a GA ${ }^{(\mathrm{x})}$ shaped molecule, and it

508 forms when the unstructured loop that LEU323 is attached to becomes part of a partial helix.

509 This releases helix B of GID1 to tilt outward as it lacks interaction with that loop (see Fig S2).

510 This motion appears as a natural breathing motion within GID1 when the DELLA protein is

511 absent. It is worth mentioning that based on structural similarities, this cleft might be the remnant

512 of the clade IV carboxylesterases (CXE) binding pocket in ancient plants (36). Assisting

513 interactions with HIS1 19 allow the $\mathrm{GA}^{(\text {a) }}$ ligands to twist, having the alkyl backbone face

514 LEU323 and the aliphatic residues adjacent. These events were observed as essential dynamics

515 of this localized region based on a principal component analysis. In Figures S3, S4, S6, and S7

516 we compare the influence of DELLA binding on these essential motions (37). From analysing

517 the pocket residues that contact the ligand when fully docked, it is likely that key interaction to

518 TYR322 prevent the LEU323 loop from opening up and allowing a facile egression. This

519 tyrosine may also be important for bioactivity because it directly interacts with the key hydroxyl

520 moiety on $\mathrm{C} 3$ of GA.

521 Based on the observation that the cleft opening is relatively large with a shorter passage to the

522 binding pocket compared to the narrower and longer channel passage, we suggest GA ${ }^{(\mathrm{x})}$ binding

523 to GID1A through the cleft in absence of DELLA protein is a significantly more rapid process

524 than through the channel. The GAI is assumed to bind to $\mathrm{gG}$ with little distinction in $\mathrm{GA}^{(\mathrm{x})}$.

525 Although it has been shown that mutations in GID1B can be made to facilitate GAI binding

526 without the presence of any GA, this has not been observed in nature, suggesting the role of GA 
527 is to act as a cofactor to facilitate the gDG complex to form. In other words, it is not the case that

528 an aDG complex cannot be stable, but rather, it is important for biological function to have the

529 presence of GA as a facilitating condition in order to control growth regulation in a plant.

530 Furthermore, $\mathrm{GA}^{(\mathrm{x})}$ dissociates from $\mathrm{gDG}$ on a much faster time scale than GAI can dissociate

531 from GID1, implying there will be some degree of binding of DELLA to GID1 in the absence of

532 GA.

\section{Proposed rectification mechanism that can select for bioactivity}

534 We propose a novel kinetic model based on the newly found cleft pathway that also takes into

535 account the role played by the DELLA N-terminus that includes three MoRFs. Our kinetic

536 model elucidates how this system recognizes bioactive GA variants under competitive binding.

537 Furthermore, we performed a phylogenetic analysis that shows there is conservation of key

538 residues within the binding pocket, cleft and channel pathways - suggesting that the same

539 general mechanism in the GAI-GID1A interaction will apply to all DELLA · GID1 complexes.

540 There is uncertainty in whether a particular GA is bioactive for a given receptor. Therefore, we

541 introduce a more appropriate language to describe GA variants in terms of major and less active

542 forms of $\mathrm{GA}^{(\mathrm{x})}$ relative to a given receptor. Subject to a wide spectrum of activities, $\mathrm{GA}^{(\mathrm{a})}$

543 denotes a subset of major active forms, while $\mathrm{GA}^{(\mathrm{i})}$ denotes a subset of less active forms. For

544 simplicity of this discussion, the two subsets represent extremes in the activity spectrum for the

545 GID1A receptor (active versus inactive). The rank order of GA variants from most bioactive to

546 inactive would likely be different with respect to a different receptor. If the sequence of another

547 receptor is highly conserved with respect to GID1A in the binding pocket as well as the two

548 binding pathways, then the rank ordering of GA activity will presumably be similar. To 
549 emphasise generality of the kinetic model, we discuss its components in terms of the GAI and

550 GID1 family of proteins.

551 Our results suggest that a molecule resembling the columbic and geometrical properties of $\mathrm{GA}^{(\mathrm{x})}$

552 can bind into GID1, particularly via the cleft pathway. Notably this includes GA variants that are

553 often only described as "inactive" or "intermediates" in the literature. Therefore, a degree of

554 caution should be exercised when classifying $\mathrm{GA}^{(\mathrm{x})}$ as bioactive or not. With this in mind it is

555 axiomatic that recognition of bioactivity must involve GID1 in some capacity. If GID1 can bind

556 an array of $\mathrm{GA}^{(\mathrm{x})}$ that likely includes both bioactive and inactive examples, then, this suggests a

557 mechanism of action removes inactive (or less active) forms of GA from the receptor or affect

558 the DELLA protein binding in other ways. Differentiation between $\mathrm{GA}^{(\mathrm{a})}$ and $\mathrm{GA}^{(\mathrm{i})}$ can occur

559 before and/or after DELLA is bound to the gG system. As shown in Figure 6, we construct a

560 generic 1 st order kinetic model for a proposed rectification process.

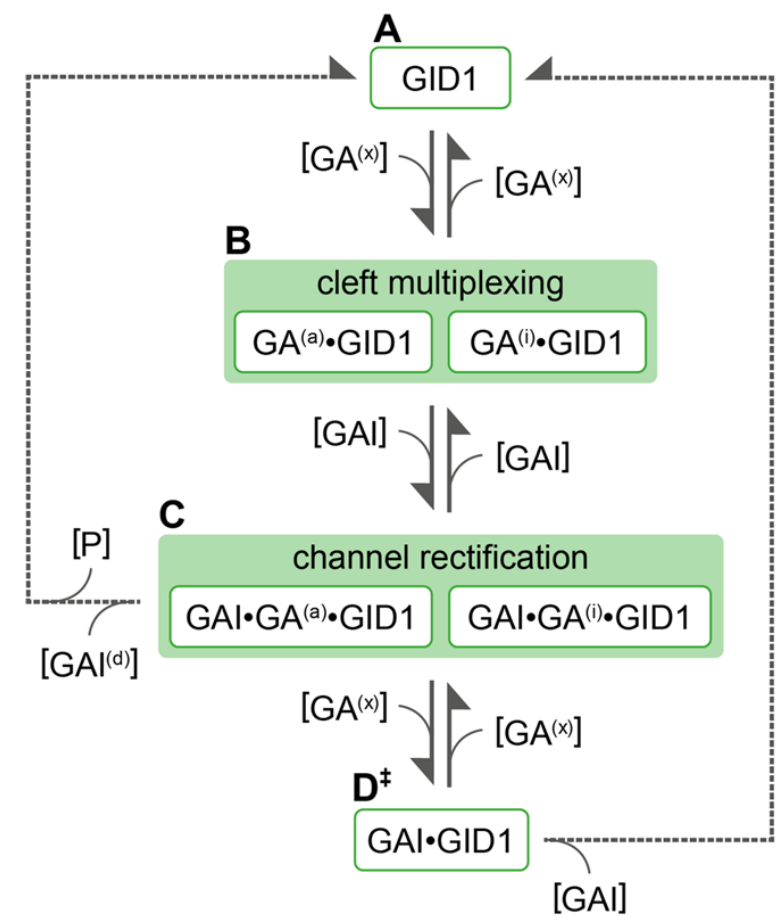


563 The $\mathrm{GA}^{(\mathrm{x})}$ in Figure 6 refers to any GA variant, but once a GA variant interacts with GID1, it

564 then becomes possible to classify the GA as a major or less active form. As a simplification, only

565 the extremes are tracked to show the possibility for competition between active and inactive

566 forms of GA, denoted as $\mathrm{GA}^{(\mathrm{a})}$ and $\mathrm{GA}^{(\mathrm{i})}$ respectively. This simplification demonstrates salient

567 features of the model by considering kinetic rates for only two types of GA (active and inactive),

568 but in reality, different kinetic rates will likely apply to each GA variant comprising a broad

569 spectrum of activities.

570 In state A, we have a free GID1 in an environment containing a mixture of GA variants, where

571 GID1 has opportunity to bind to any GA variant, represented by $\mathrm{GA}^{(\mathrm{x})}$. Since the open cleft does

572 not have a high level of specificity for the type of $\mathrm{GA}^{(\mathrm{x})}$ to bind, the cumulative concentration of

573 all GA variants drives GA association to state B. The GA · GID1 complex is now primed to bind

574 with GAI. Since GAI association is a relatively slow process, it is expected that many cycles of

575 association and dissociation of different GA variants through the open cleft in GID1 occurs in

576 the interim. The key role of the cleft opening is to multiplex the kinetics of all $\mathrm{GA}^{(\mathrm{x})}$ associations

577 that can take place to populate state B while depleting state A as much as possible depending on

578 a variety of details. While details depend on environmental conditions such as concentration of

579 repressor, receptor, protease, and GA variants, the qualitative features remain robust. Eventually,

580 GAI binds to GID1 to form the GAI $\cdot$ GA $\cdot$ GID1 complex that defines state C.

581 The details of which GA variant is bound affects the stability and conformational dynamics of

582 the GAI - GA - GID1 complex, which in turn affects the propensity for the E3 ubiquitin ligase to

583 exercise proteolysis to degrade GAI. The GAI $\cdot \mathrm{GA}^{(\mathrm{a})} \cdot \mathrm{GID} 1$ complex is primed for GAI to

584 undergo degradation, but the GAI $\cdot \mathrm{GA}^{(\mathrm{i})} \cdot \mathrm{GID} 1$ complex with less active GA varaints is

585 unsuitable, being the bottleneck that ultimately differentiates the bioactivity across GA variants. 
586 Dissociation of GA variants is possible through the closed cleft (the cleft is blocked by GAI) and

587 the open channel within GID1. Since the time scale of GAI dissociation is relatively long

588 compared to GA dissociation and association from the binding pocket in GID1, it is possible to

589 reach state D.

590 State D is not stable. Eventually the unstable GAI - GID1 complex will dissociate because a GA

591 variant is necessary to maintain its stability. However, this dissociation process takes place on a

592 time scale that is much longer than the time scale for GA variant association, mainly through the

593 channel. The rates of dissociation and association will be nuanced depending on the moieties of

594 the GA variant and the sequence of GID1, and especially the structure and dynamics of the GAI

$595 \cdot$ GID1 complex that also depends on the sequence of GAI (or indeed other DELLA proteins

596 where they exist). The upshot of these details is that the overall dissociation rates for GA ${ }^{(i)}$ are

597 greater than $\mathrm{GA}^{(\mathrm{a})}$ and the overall association rates for $\mathrm{GA}^{(\mathrm{a})}$ are greater than $\mathrm{GA}^{(\mathrm{i})}$ in the kinetics

598 that bridge states C and D.

599 A kinetic equilibrium is set up that drives the concentration ratio of $\sum_{a}[\mathrm{GA}(\mathrm{a})]$ to $\sum_{x}[\mathrm{GA}(\mathrm{x})]$

600 toward 1 . This result is insensitive to model details (i.e. the rates) provided the general trends

601 described above are satisfied. As a consequence, rectification of less active GA variants takes

602 place to amplify the propensity of the biologically important GAI $\cdot \mathrm{GA}^{(\mathrm{a})} \cdot \mathrm{GID} 1 \mathrm{complex}$. That

603 is, the GAI - GA ${ }^{\text {(a) }} \cdot$ GID1 complex will be well populated even within an environment where the

604 ratio of $\sum_{a}[\mathrm{GA}(\mathrm{a})]$ to $\sum_{x}[\mathrm{GA}(\mathrm{x})]$ for the mixture of GA variants in solution is small. An

605 amplification factor, defined by the ratio of $\sum_{a}[\mathrm{GA}(\mathrm{a})]$ to $\sum_{x}[\mathrm{GA}(\mathrm{x})]$ within the binding pocket

606 divided by the ratio of $\sum_{a}[\mathrm{GA}(\mathrm{a})]$ to $\sum_{x}[\mathrm{GA}(\mathrm{x})]$ for the GA variant mixture can easily reach up

607 to 10. 
608 It is also possible that the dissociation rate for GAI from the GAI $\cdot \mathrm{GA}^{(i)} \cdot \mathrm{GID} 1$ complex is

609 greater than the dissociation rate for GAI from the GAI $\cdot \mathrm{GA}^{(\mathrm{a})} \cdot \mathrm{GID} 1$ complex. This is another

610 nuanced way rectification can take place. We note that this would be a significantly slower

611 process than $\mathrm{GA}^{(\mathrm{i})}$ leaving the complex. Our data suggest both types of nuanced mechanisms can

612 work in tandem. If both mechanisms are present to rectify the less active GA in the binding

613 pocket, amplification factors near 100 can be obtained. Since we cannot offer any

614 biophysical/chemical rationale for why these proposed mechanisms would not be present, we

615 propose that it is through protein evolution that repressors and receptors become in tune with one

616 another through a specific set of GA variants. Given the simplicity in the explanation of how a

617 repressor-receptor pair functions with high fidelity, while making use of a large diverse set of

618 GA growth hormones, it is fitting to look at evolutionary consequences in plant biology.

619 Implications for evolution of the Gibberellin signalling system

620 The high degree of conservation over the 37 GA contact residues across the four main GID1

621 groups suggest that most of the conclusions we drawn about the ligand migration pathway from

622 our analysis of GID1A are likely to be true for most or all other GID1 proteins. Out of this set of

623 residues there were only two residues in the channel pathway that show a significant degree of

624 substitutions that are not necessarily conservative in nature. The proposed kinetic model is quite

625 plastic, where we suggest that the control of major and minor active forms of GA is best

626 understood by an evolutionary perspective.

627 One of the more variable sites among the contact residues is an ASN residue 32 in GID1A that

628 undergoes H-bonding with alcohol moieties of all bioactive GA during channel egressions and

629 that can change to other polar residues (HIS, LYS, THR) or even a non-charged MET in basal

630 species. A more detailed examination of these different forms of GID1 would be required to 
631 ascertain if it is likely that these changes could significantly change the behaviour of one or more

632 GA variant as it passes through the channel. This residue is part of alpha helix B which appears

633 to play a significant role in the receptor "breathing" motion that we discuss above. We suggest

634 these variations can have significant impact on different versions of GID1 e.g. they may have

635 somewhat different specificities for different GA variants or interact differently with the DELLA

636 protein that also interacts with this alpha helix. Interestingly this ASN residue appears not to be

637 contacted by many of the $\mathrm{GA}^{(\mathrm{i})}$ variants for which there is no convincing data for bioactivity.

638 This could suggest the potential for differences in this "breathing" motion in some GID1 loci

639 associated with particular GA variants and perhaps even a lack of bioactivity in the context of a

640 particular gDG complex.

641 The second contact residue showing a significant amount of non-conservative substitutions is a

642 THR residue in GID1A-240 that was also identified by Hao et al. as a key GA contact residue

643 and is a residue that apparently undergoes hydrogen bonding with GA lactone. This residue

644 partners with ASP243 to initiate the channel pathway before GA ${ }^{\mathrm{x}}$ rotates to interact with

645 TYR247 and ASP243. The variability here seems to be large confined to the two dicot subgroups

646 (GID1A and GID1B). This THR residue site displays substitutions to LYS, ARG and GLY

647 which could well have major implications for H-bonding to one or more GA variant and being at

648 the start of the pathway this could significantly impact on the rate of engagement of this pathway

649 by different $\mathrm{GA}^{(\mathrm{x})}$ forms for GID1 variants carrying these substitutions.

650 The dicots are unusual in showing a very deep phylogenetic split into two major GID1 clades.

651 Monocots generally contain just one GID1 locus or, if there has been any duplication, these are

652 likely to be more recent events. The presence of two GID1 clades in dicots that have been

653 evolving separately (but present together for a long time) would provide opportunities for 
654 specialized functions to evolve between these clades. Possible areas of partition of components

655 of their combined function could include specialization for different GA variants, different

656 degrees of GA signal transmission, or perhaps forming specialized interactions with a subgroup

657 of DELLA proteins. It is notable that there are often multiple members in the DELLA family in

658 dicot plant species as well.

659 In plant taxa where there is only one GID1 this specialization would not be possible and the

660 residue of choice may be more of a compromise - perhaps explaining why this residue is highly

661 conserved in the monocot and basal plant lineages and that there is a tendency for just one

662 partnering DELLA locus to exist in monocots as well. Perhaps the ability of some GID1B

663 proteins to interact with DELLA proteins in the absence of GA ligand (albeit it usually less

664 strongly than in the presence of GA), similarly reflects some of this specialization of function. In

665 addition to the major divide between GID1A and GID1B there have been a number of

666 subsequent duplication events in both of these clades that predate the split between major

667 divisions within the Asterids and Rosids. We suggest that a very early duplication event in the

668 dicots released additional evolutionary potential for variation to occur without a loss of function.

669 As a result, dicots may have taken advantage of this to evolve a more complex mix of GID1 and

670 partnering DELLA loci. We would therefore expect there to be a higher degree of specialization

671 in these loci not possible in monocots and basal plant species. The higher variability in two GA ${ }^{(a)}$

672 contact residues in the channel pathway of dicots may also reflect this higher propensity for

673 specialization in dicots. 
675 Based on data from simulation, experiment, and evolutionary analyses, we put forth the most

676 complete kinetic model for how gibberellin promotes plant growth by enhancing binding

677 between GAI repressors and GID1 receptors to regulate GAI degradation and control plant

678 growth by de-repressing GA response genes. There are two binding pathways in the form of a

679 cleft and channel in GID1. The cleft pathway is open when GAI is not present, giving easy

680 accessibility to GA variants to bind within the binding pocket. The GA variants acting as

681 cofactors help GAI bind to GID1. After the formation of this complex, if the GA variant is

682 bioactive, a subsequent process for the degradation of GAI eventually follows. However, there is

683 a continual kinetic process that takes place with GA variants leaving and re-entering the binding

684 pocket. Due to large differences in time scales, many cycles of GA exchanges can take place

685 before the GAI - GID1 complex has time to dissociate, which is inevitable if no GA re-enters the

686 binding pocket. As major and less active forms of GA enter and exit the binding pocket

687 recurrently with disparate rates that favour the major active forms, rectification of less active

688 forms occurs to yield higher propensity for a major active form to control growth.

689 The biological ramification of this kinetic model partly accounts for the highly diverse set of GA

690 variants known to be present in plants. Within the cellular environment there will be a mixture of

691 GA variants available to bind to GID1 through competitive binding. In the initial binding of GA

692 to GID1, the consequence of binding of major active or less active forms of GA is similar. The

693 binding of GAI to GID1 will be enhanced for almost all GA variant. The interactions between

694 GAI and GID1 in the GA - GAI - GID1 complex further promotes specificity. If the captured GA

695 is a major active form, the degradation of GAI can take place via the known SLY1 facilitated

696 ubiquitination and proteolysis. Otherwise, the rectification process takes place, which is 
697 especially needed in cases where the concentration of the major active form is considerably less

698 than the combined set of less active forms. This mechanism allows plants to readily sense fine

699 concentration changes of bioactive GA molecules with high specificity to enable certain GA

700 variants to trigger specific signalling events. In this scenario, the large number of different GA

701 variants found in plants becomes biologically advantageous for homeostasis and/or specialisation

702 when combined with different DELLA · GID1 complexes (particularly in dicots). Our proposed

703 mechanism is consistent with the general paradigm of protein-ligand binding and protein

704 evolution. Overall, passive specificity is enabled by small changes in pathways of GID1, which

705 gives rise to the complex series of interactions with the disordered DELLAs in a variety of plants

706 which enables their complex growing habits.

707 Methods

708 Computational modelling and analysis

709 The Murase et al. (27) resolved structure from A. thaliana GA receptor GID1A and the N-

710 terminal fragment of the GAI conserved across various plant species (PDB Accession no: 2ZSH)

711 was partially unresolved. This is explained by the inherent disordered nature of the GAI N-

712 terminal structure (22). The absence of large parts of the disordered domains prevent realistic

713 simulation of this hormone receptor system. Therefore, missing segments were constructed using

714 I-TASSER (Iterative Threading ASSEmbly) (28) ab-initio and threading method software. The

715110 residue N-terminal DELLA sequence from the $2 \mathrm{ZSH}$ structure was used to generate a more

716 complete model by filling in the missing components between the three MoRF segments that

717 were partially predicted in $2 \mathrm{ZSH}$. 
718 The DELLA structure represents a designed subsection of the DELLA GAI sequenced used in

719 the source study. As such the source material sequence published with 2ZSH (chain B) is used to

720 number these residues (1-110). The GID1A (chain A) structure in this model is missing 5

721 residues from the N-terminal and 2 from the C-terminal ends. Both 2ZSHA and 2ZSHB have a

722 short remnant of an artificial HRV-3C protease cleaved His-tag at the N-terminus. For GID1A

723 the residue numbering includes these missing residues as aligned from uniport Arabidopsis

724 thaliana sequence for GID1A (UniProtKB: Q9MAA7-1). We exclude residues in the tag

725 remnant from further discussion.

726 Ten full atomistic models of the DELLA disordered protein were generated from residues 1 to

727 110. Variation in the top models at the disorder loops showed minor orientation differences. The

728 top scoring model was checked by potential energy calculations using ProSA-web (38). ProSA is

729 based on PDB structures derived from X-ray diffraction (XRD) and Nuclear Magnetic

730 Resonance (NMR) based PDB structures to score unseen structures. The deduced DELLA

731 structure is consistent in conformation to NMR structures and consistent with Ramachandran

732 backbone angles. To arrive at the gDG system, the DELLA GAI N-terminus was placed by a

733 structural alignment to the original fragments of the DELLA subunit. Furthermore, each GA(x)

734 ligand was aligned to the $2 \mathrm{ZSH}$ structure before minimization for both $\mathrm{gDG}$ and $\mathrm{GA}^{(\mathrm{x})} \cdot \mathrm{GID} 1 \mathrm{~A}$

735 (gG) systems. All GA that had carboxylic C6 were deprotonated before calculating ligand

736 parameters or docking into GID1A (39). All simulations were carried out on Linux based high

737 performance computing clusters.

738 The GA variants were optimized in AMBER using semi-empirical BCC available inside the

739 Ambertools software (40), then the ligands were aligned with $\mathrm{GA}_{3}$ of the crystal 2ZSH before

740 minimizing the system in NAMD. In each system solvent within $5 \mathrm{~nm}$ of the $2 \mathrm{ZSH} \mathrm{GA}_{3}$ was 
741 kept and placed back into the structure before energy minimization as defined in Kokh et al.

742 (31). The steps performed in this process consisted of the preparation of the solvated protein-

743 ligand complex, followed by a 20 ns production run, or a steady state simulation, of water and

$744 \mathrm{NaCl}$ ions at $0.5 \mathrm{mmol}$ with the various systems studied at $300^{\circ} \mathrm{K}$, followed by multiple tRAMD

745 simulations in NAMD to obtain ligand dissociation trajectories. The tRAMD relative residence

746 times were obtained using a previously reported protocol (31) adapted to Matlab using

747 PDFEstimator (41) in Matlab 2020a. Systematic evaluation of force constants found that at a

748 force of $20 \mathrm{kcal} \mathrm{mol}^{-1} \AA^{-1}$ allowed egression to a threshold of $30 \mathrm{~nm}$ from the docked position in

749 a computationally tractable timespan. Similar force evaluation was performed for the egressions

750 of the entire DELLA protein. A significantly higher force of $80 \mathrm{kcal} \mathrm{mol}^{-1} \AA^{-1}$ was found to allow

751 egression on $\mathrm{ns}$ time scale to reach a distance of $30 \mathrm{~nm}$ from the original position of DELLA

752 chain.

753 Visual Molecular Dynamics (VMD) analysis of trajectories was performed using in-house

754 designed scripts in TCL. A cut-off distance of $3.5 \AA$ and angle of $30^{\circ}$ for hydrogen $(\mathrm{H})$ bond

755 counting. Hydrophobic contacts were defined using known nonpolar residues and a contact cut-

756 off distance of $3.5 \AA$ for conservative estimates of contacts. These were tabulated into a text file

757 for further processing. Raw counts of interactions to residues from a defined reference set were

758 used as samples in statistical analysis. Significance of differences were selected from a rational

759 p-value threshold of 0.05. Recurrent residue sets were simple logical comparisons between lists

760 of interaction residues between various simulation sets. For recurrent residues in various

761 important comparisons the active GA subsets were used to create a residue subset, which was

762 then compared to inactive GA interaction lists. 


\section{Phylogenetic analysis of Gibberellin signalling components}

764 We subjected both the N-terminus of DELLA proteins (up to but not including the GRAS

765 domain) and the GID1 receptor family to a similar phylogenetic analysis in order to determine if

766 residues of interest from the above analyses were broadly conserved or variable. The degree of

767 variation within the disordered segments of the DELLA family N-terminus in particular only re-

768 enforced that the three MoRF regions identified previously by analysis of a smaller dataset (4)

769 were highly conserved. Most of the residues identified in the above modelling analysis were

770 present in these conserved MoRF motifs. As this phylogeny has little new significant data, it is

771 not presented. In contrast the GID1 protein family is clearly much more highly conserved across

772 its entire protein sequence. Gene trees developed under Plant Compare of EnsemblPlants were

773 used as a basis for obtaining the orthologs of the GID1 family of proteins from all plant taxa and

774 their basal ancestors (Basal Angiosperms like Amborella genus and Lesser club moss sequences

775 from Selaginella moellendorffii). There are no sequences in the more basal Earth mosses or

776 liverworts (Physcomitrella and Marchantia genera). This tree contains four major subclade

777 branches.

778 The members of four main subclade branches (basal plants and mosses, monocots and two major

779 dicot subclades) were further split into major divisions at the suborder (monocot) and superclass

780 (dicot) levels, combined with evidence for inferred duplication events in the GID1A and GID1B

781 clades. Based on this analysis the dataset was divided into 26 groupings with 1 to 22 member

782 proteins. The members of each grouping were then downloaded as a set of aligned groupings into

783 Geneious, given a new nomenclature to identify the groupings and realigned in Geneious with

784 default settings. 
785 These alignments were examined for proteins with large gaps in the core of the GID1 sequence

786 and N- or C-terminal extensions. Proteins with large deletions in the core were completely

787 deleted from the subsets as these are likely to be miss-annotated and this will affect the

788 conservation level of residues in the deleted regions. Large unique N- and C-terminal extensions

789 were removed from the alignments as they result in extensions with apparent "perfectly

790 conserved" protein regions due to the lack of other aligned sequences - thus leading to

791 misleading conservation information. A consensus sequence set at $90 \%$ conservation was derived

792 from each of the modified 26 groupings, and named via the new nomenclature. These consensus

793 sequences were then re-aligned as subtree alignments and also the full set of all plant consensus

794 proteins.

795 The aligned sequences are ordered with the most basal consensus group at the bottom and more

796 derived consensus groups (as inferred from the EnsemblPlants tree) progressively aligned

797 towards the top of the alignment. The letter $\mathrm{X}$ in these alignment denotes a lack of conservation

798 at $90 \%$ for the particular group at that site whereas a residue means greater than $90 \%$

799 conservation. It should be noted that many alignments had fewer than 10 members and some

800 (with only 1 member) "appear" to be perfectly conserved in the alignment but this is an artefact

801 of the number of group members.

802 Next we utilised this phylogenetically "collapsed" consensus alignment in Figure S10 to examine

803 each of the key GID1A residues identified in the modelling analysis in the binding pocket, cleft

804 and channel to asses if it was identified as a conserved residue across all plant lineages,

805 conserved across one or more of the four major subclades, conserved across one or more of the 
806 subgroupings with these subclades or variable across all 26 divisions. The results of this analysis

807 are presented in supplementary information tables $3,4,5$.

808 Supplemental Information

809 Figure S1: RMSD GAI MoRFs during production runs. RMSD from production runs of GAI

810 MoRF subsets of gDG and aDG systems. Computed from trajectories corrected for PBC via

811 VMD. RMSD frames were averaged over intervals of 300 frames, standard deviation shown in

812 bars.

813 Table S1: Logic table for Channel pathway egressions in gG system. H-bond and Contact

814 residues during Channel pathway egressions gG.

815 Table S2: Logic table for Cleft pathway egressions in gDG system. H-bond and Contact

816 residues during cleft pathway egressions gDG, * interacting GAI residue close to the DELLA

817 motif.

818 Figure S2: Initial and final frame for Cleft egression in gG system. (A) Initial

819 (green/transparent) and final (cyan/solid surface) frames in ribbon display mode from a cleft

820 egression in GID1A with $\mathrm{GA}_{3}$. The slight flex or "breathing" motion of helix B, shown in red, in

821 GID1A allows a clear path. (B) Initial (light shading) and final (darker shading) frame of space

822 filling models of the pocket subset (Table 1) showing a cleft egression in GID1A and

823 flexing/breathing of helix $\mathrm{B}$ as $\mathrm{GA}_{3}$ leaves the cleft. 
824 Figure S3: RMSF mapped cartoon of GA 3 DELLA.GID1A egression pathways. JEDi RMSF

825 maps from example egression paths in GA 3 -DELLA-GID1A system. Perspective of helix B

826 GID1A and DELLA MoRF1.

827 Figure S4: RMSF mapped cartoon of GA $\mathbf{H}_{3}$ GID1A egression pathways. JEDi RMSF maps of

828 examples egression paths in $\mathrm{GA}_{3}$ GID1A system. Perspective of GID1A N-terminal in front.

829 Figure S5: RMSD of $\mathbf{G A}^{(\mathbf{x})}$ in $\mathbf{g G}$ system. RMSD of GA ligand in gG systems during $20 \mathrm{~ns}$

830 production run.

831 Figure S6: Essential motion PCA weights tRAMD for GA3-DG complex. PCA performed at

832 alpha carbon level for GA3-GID1-DELLA tRAMD egressions for examples of the two

833 pathways, first PCA mode showing. Residues numbered linearly from GAI-1 to GID1A-450, see

834 methods for numbering correction. The channel pathway is plotted in red and the cleft pathway

835 in blue. Note that residues 110-450 in this Figure match residues 0 to 340 in SI Figure 7 below.

836 Figure S7: Essential motion PCA weights tRAMD for GA3-GID1A complex. PCA performed

837 at alpha carbon level of GA3-GID1A tRAMD egressions for examples of the two pathways. The

838 channel pathway data are plotted in red and the cleft pathway data in blue. Note the y-axis

839 change due to the loss of dynamics in GID1A cleft egression.

840 Figure S8: DELLA RMSF comparison between Apo, GA ${ }^{(a)}$ and GA ${ }^{(i)}$ complexes with GID1.

841 RMSF at alpha carbon level for DELLA subset of gDG (red line with $\mathrm{GA}^{(\mathrm{i})}$, black line with

$842 \mathrm{GA}^{(\mathrm{a})}$ ) and DG (apo-pink line) systems. 


\section{Figure S9: RMSF analysis for GID1A in binary and tertiary complexes with DELLA and}

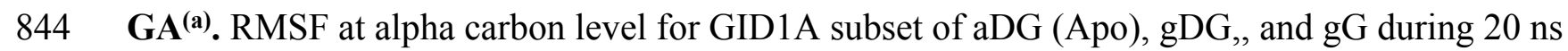

845 production run for $\mathrm{GA}^{(\mathrm{a})}$ containing systems. gDG and $\mathrm{gG}$ systems are averaged with standard

846 deviation shown as bars.

\section{Table S3: Analysis of contact residues in the binding pocket of GID1 across four main}

848 phylogenetic divides. * 1 - Residues underlined indicate H-bond contacts, the others indicate

849 hydrophobic interactions. *2 - denotes residues also identified as important in GA binding by

850 Hao et al. (2013). Frequent (in capitals) and infrequent (in lower case) residue variants are given

851 in brackets using the single letter amino acid code-note the lower case $\mathrm{L}$ is underlined to

852 distinguish it from upper case i. $\mathrm{C}=$ highly conserved across this clade (in bold indicates a

853 conserved but different residue to that found in the GID1A template), cases of minor variation

854 are given in brackets; $\mathrm{S}=$ mainly conserved with conservative substitutions only across this clade.

Table S4: Analysis of channel contact residues across the main phylogenetic divides of

856 GID1. * 1 - Residues underlined indicate H-bond contacts, the others indicate hydrophobic

857 interactions. *2 -denotes residues also identified as important in GA channel by Hao et al.

858 (2013). Frequent (in capitals) and infrequent (in lower case) residue variants are given in

859 brackets using the single letter amino acid code-note the lower case $\mathrm{L}$ is underlined to distinguish

860 it from upper case i. $\mathrm{C}=$ highly conserved across this clade (in bold indicates a conserved but

861 different residue to that found in the GID1A template), cases of minor variation are given in

862 brackets; $\mathrm{S}=$ =mainly conserved with conservative substitutions only across this clade; $\mathrm{V}^{*}=$

863 variable. 
Table S5: Cleft contact residue conservation across 4 main phylogenetic divides of GID1.

$865 * 1-$ Residues underlined indicate H-bond contacts, the others indicate hydrophobic interactions.

866 Frequent (in capitals) and infrequent (in lower case) residue variants are given in brackets using

867 the single letter amino acid code-note the lower case L is underlined to distinguish it from upper

868 case i. $\mathrm{C}=$ highly conserved across this clade (in bold indicates a conserved but different residue

869 to that found in the GID1A template), cases of minor variation are given in brackets; $\mathrm{S}=$ mainly

870 conserved with conservative substitutions only across this clade.

871 Figure S10: Alignment of 26 GID1 plant and basal plant subclades represented by $90 \%$

872 conserved consensus. Geneious alignment of consensus GID1 sequences (created at 90\%

873 identity) from 26 sub-clades defined by duplication nodes in plant classification subdivisions in

874 the EnsemblPlants database. All letters denote single letter amino acid code with the exception of

$875 \mathrm{X}$ and $\mathrm{Z}$. X denotes residues not conserved in the relevant consensus sequence, $\mathrm{Z}$ denotes

876 conserved charged residues at this site. The clade naming convention includes an abbreviated

877 form of the plant family before the GID1 protein name, a major division into GID1A or GID1B

878 in dicot families or indication the clade is a monocot (mon) or more basal (bas) plant family and

879 a final letter (A, B or C) to distinguish multiple subclades with distinct evolutionary histories in

880 the same plant family. The subclades are organised by the most basal subclades at the bottom

881 followed by monocot specific clades, then the GID1B subclades and ending with the GID1A

882 dicot subclades at the top of the alignment to match the tree like phylogeny structure. Moss-

883 Slagenella sp. sequences; Ambt-Amborella trichopoda sequence; Dios-Dioscorea sp. sequences

884 (yam family); Zing-Zingiberales order sequences from Musa sp.; Pani-Panicoideae sub-families

885 within the Poaceae family ; Pooi -Pooideae sub-families within the Poaceae family; Oryz -

886 Oryzideae sub-families within the Poaceae family; Cary-Beta sp. sequences in the 
887 Caryophyllales order; Lami- Lamiid subclade sp. sequences in the Euasterid clade; Camp-

888 Campanulid subclade sp. sequences in the Euasterid clade; Eric-Ericales order sp. sequences;

889 Vital-Vitales order sequences from Vitis in the SuperRosid clade; Malv- Malvid clade sequence

890 in the Eurosid clade; Fab- Fabid clade sequence in the Eurosid clade.

891 Movie S1: PCA motions. (MP4) The intrinsic dynamics based on Principal Component

892 Analysis associated with PCA mode 1 is shown for the gDG and gG systems when GA3 is

893 bound. The rendering does not include the GA3 molecule. Two movies are shown for the gDG

894 system. The "front" view corresponds to the channel in view, while the "back" view corresponds

895 to the cleft in view. Likewise, two movies are shown for the gG system to show the front and

896 back views. The ribbon rendering has the coloring scheme where blue indicates very little

897 mobility, green indicates low mobility, yellow indicates moderate mobility, orange indicates

898 considerable mobility and red indicates very high mobility.

899 Movie S2: Example GA3 egressions. (MP4) The last part of tRAMD simulations are shown for

900 the case of GA3 dissociating from the channel and cleft pathways in the gDG and gG systems. It

901 is clear there are multiple ways for GA to enter and exit the binding pocket.

902 Readme S1: Description of data and script location. (TXT)

\section{Acknowledgements}

904 This international collaboration was funded by the New Zealand MBIE (Ministry of Business,

905 Innovation and Employment) Endeavour programme grant C11X1804 to ER. The authors wish

906 to thank editorial contributions from Drs Toshi Foster and Cyril Hamiaux (Plant \& Food

907 Research). Donna Gibson (Plant \& Food Research) for rendering and conceptualizing art. We 
908 wish to acknowledge the use of New Zealand eScience Infrastructure (NeSI) high performance

909 computing facilities in this research. These national facilities are provided by NeSI and funded

910 jointly by NeSI's collaborator institutions and through the MBIE research infrastructure

911 programme (URL https://www/nesi.org.nz) and made available to other MBIE funded

912 programmes. We also acknowledge the provision of additional computing facilities and

913 resources by the University of North Carolina at Charlotte.

\section{Author contributions}

915 Conceptualization: Charles C. David, Marion Wood, Xiaolin Sun, Donald J. Jacobs, Erik H. A.

916 Rikkerink

917 Formal analysis: John Patterson, Charles C. David, Donald J. Jacobs, Erik H. A. Rikkerink

918 Funding acquisition: Marion Wood, Erik H. A. Rikkerink

919 Investigation: John Patterson, Charles C. David, Marion Wood, Xiaolin Sun, Donald J. Jacobs,

920 Erik H. A. Rikkerink

921 Project administration: Donald J. Jacobs, Erik H. A. Rikkerink

922 Supervision: Donald J. Jacobs, Erik H. A. Rikkerink

923 Validation: Charles C. David, Donald J. Jacobs, Erik H. A. Rikkerink

924 Visualization: John Patterson, Charles C. David, Marion Wood

925 Writing - original draft: John Patterson, Marion Wood, Erik H. A. Rikkerink

926 Writing - review \& editing: John Patterson, Charles C. David, Marion Wood, Xiaolin Sun,

927 Donald J. Jacobs, Erik H. A. Rikkerink 
929 1. Livesay DR, Jacobs DJ. Conserved quantitative stability/flexibility relationships (QSFR)

930 in an orthologous RNase H pair. Proteins: Structure, Function, and Bioinformatics.

$9312006 ; 62(1): 130-43$.

932 2. Verma D, Jacobs DJ, Livesay DR. Changes in Lysozyme Flexibility upon Mutation Are

933 Frequent, Large and Long-Ranged. PLOS Computational Biology. 2012;8(3):e1002409.

934 3. Li T, Tracka MB, Uddin S, Casas-Finet J, Jacobs DJ, Livesay DR. Redistribution of

935 Flexibility in Stabilizing Antibody Fragment Mutants Follows Le Châtelier's Principle. PLOS

936 ONE. 2014;9(3):e92870.

937 4. Sun X, Xue B, Jones W, Rikkerink E, Dunker A, Uversky V. A functionally required

938 unfoldome from the plant kingdom: intrinsically disordered N-terminal domains of GRAS

939 proteins are involved in molecular recognition during plant development. Plant Molecular

$940 \quad$ Biology. 2011;77(3):205-23.

941 5. Sun X, Rikkerink EHA, Jones WT, Uversky VN. Multifarious Roles of Intrinsic Disorder

942 in Proteins Illustrate Its Broad Impact on Plant Biology. The Plant Cell. 2013;25(1):38-55.

943 6. Peng J, Carol P, Richards DE, King KE, Cowling RJ, Murphy GP, et al. The Arabidopsis

944 GAI gene defines a signaling pathway that negatively regulates gibberellin responses. Genes

945 Dev. 1997;11.

946 7. Peng J, Richards DE, Hartley NM, Murphy GP, Devos KM, Flintham JE, et al. 'Green

947 revolution' genes encode mutant gibberellin response modulators. Nature. 1999;400(6741):256-

94861. 
949 8. Briones-Moreno A, Hernández-García J, Vargas-Chávez C, Romero-Campero FJ,

950 Romero JM, Valverde F, et al. Evolutionary Analysis of DELLA-Associated Transcriptional

951 Networks. Frontiers in Plant Science. 2017;8(626).

952 9. Li Y, Yang Y, Hu Y, Liu H, He M, Yang Z, et al. DELLA and EDS1 Form a Feedback

953 Regulatory Module to Fine-Tune Plant Growth-Defense Tradeoff in Arabidopsis. Molecular

954 Plant. 2019;12(11):1485-98.

955 10. Navarro L, Bari R, Achard P, Lisón P, Nemri A, Harberd NP, et al. DELLAs Control

956 Plant Immune Responses by Modulating the Balance of Jasmonic Acid and Salicylic Acid

957 Signaling. Current Biology. 2008;18(9):650-5.

958 11. Wild M, Davière J-M, Cheminant S, Regnault T, Baumberger N, Heintz D, et al. The

959 Arabidopsis DELLA RGA-LIKE3 Is a Direct Target of MYC2 and Modulates Jasmonate

960 Signaling Responses. The Plant Cell. 2012;24(8):3307-19.

961 12. Wild M, Achard P. The DELLA protein RGL3 positively contributes to

962 jasmonate/ethylene defense responses. Plant Signal Behav. 2013;8.

963 13. Qi T HH, Wu D, Yan J, Qi Y, Song S, Xie D () Arabidopsis DELLA and JAZ proteins

964 bind the WD-repeat/bHLH/MYB complex to modulate gibberellin and jasmonate signaling

965 synergy. . Plant Cell 2014;26(3):1118-33.

966 14. Zentella R, Sui N, Barnhill B, Hsieh WP, Hu J, Shabanowitz J, et al. The Arabidopsis O-

967 fucosyltransferase SPINDLY activates nuclear growth repressor DELLA. Nature chemical

968 biology. 2017;13(5):479-85.

969 15. Gallego-Bartolomé J, Alabadí D, Blázquez MA. DELLA-Induced Early Transcriptional

970 Changes during Etiolated Development in Arabidopsis thaliana. Plos One. 2011;6(8):e23918. 
971 16. Zhang Y, Zhang Y, McFarlane HE, Obata T, Richter AS, Lohse M, et al. Inhibition of

972 TOR Represses Nutrient Consumption, Which Improves Greening after Extended Periods of

973 Etiolation. Plant Physiology. 2018;178(1):101-17.

974 17. Nir I, Shohat H, Panizel I, Olszewski N, Aharoni A, Weiss D. The Tomato DELLA

975 Protein PROCERA Acts in Guard Cells to Promote Stomatal Closure. The Plant Cell.

$976 \quad 2017 ; 29(12): 3186-97$.

977 18. Nohales MA, Kay SA. GIGANTEA gates gibberellin signaling through stabilization of

978 the DELLA proteins in Arabidopsis. Proceedings of the National Academy of Sciences.

$979 \quad 2019 ; 116(43): 21893-9$.

980 19. Li S, Tian Y, Wu K, Ye Y, Yu J, Zhang J, et al. Modulating plant growth-metabolism

981 coordination for sustainable agriculture. Nature. 2018;560. (7720):595-600.

982 20. Wild M, Davière J-M, Regnault T, Sakvarelidze-Achard L, Carrera E, Lopez Diaz I, et

983 al. Tissue-Specific Regulation of Gibberellin Signaling Fine-Tunes Arabidopsis Iron-Deficiency

984 Responses. Developmental Cell. 2016;37(2):190-200.

985 21. Salanenka Y, Verstraeten I, Löfke C, Tabata K, Naramoto S, Glanc M, et al. Gibberellin

986 DELLA signaling targets the retromer complex to redirect protein trafficking to the plasma

987 membrane. Proceedings of the National Academy of Sciences. 2018;115(14):3716-21.

988 22. Ross JJ, Miraghazadeh A, Beckett AH, Quittenden LJ, McAdam EL. Interactions

989 between gibberellins and other hormones. Annual Plant Reviews, Volume 49: John Wiley \&

990 Sons, Ltd; 2016. p. 229-52.

991 23. Davière J-M, Achard P. A Pivotal Role of DELLAs in Regulating Multiple Hormone

992 Signals. Molecular Plant. 2016;9(1):10-20. 
993 24. Thomas SG, Blázquez MA, Alabadí D. Della Proteins: Master Regulators of Gibberellin-

994 Responsive Growth and Development. Annual Plant Reviews, Volume 49: John Wiley \& Sons,

995 Ltd; 2016. p. 189-228.

996 25. Sun XL, Jones WT, Harvey D, Edwards PJB, Pascal SM, Kirk C, et al. N-terminal

997 Domains of DELLA Proteins Are Intrinsically Unstructured in the Absence of Interaction with

998 GID1/GA Receptors. Journal of Biological Chemistry. 2010;285(15):11557-71.

999 26. Sun T-P. The Molecular Mechanism and Evolution of the GA-GID1-DELLA Signaling

1000 Module in Plants. Current Biology. 2011;21(9):R338-R45.

1001 27. Murase K, Hirano Y, Sun T-p, Hakoshima T. Gibberellin-induced DELLA recognition

1002 by the gibberellin receptor GID1. Nature. 2008;456(7221):459-63.

1003 28. Hao G-F, Yang S-G, Yang G-F, Zhan C-G. Computational gibberellin-binding channel

1004 discovery unraveling the unexpected perception mechanism of hormone signal by gibberellin

1005 receptor. Journal of Computational Chemistry. 2013;34(24):2055-64.

1006 29. Yamamoto Y, Hirai T, Yamamoto E, Kawamura M, Sato T, Kitano H, et al. A Rice gid1

1007 Suppressor Mutant Reveals That Gibberellin Is Not Always Required for Interaction between Its

1008 Receptor, GID1, and DELLA Proteins. The Plant Cell. 2010;22(11):3589-602

1009 30. Hedden P, Thomas SG. Gibberellin biosynthesis and its regulation. Biochemical Journal.

$1010 \quad 2012 ; 444: 11-25$.

1011 31. Kokh DB, Amaral M, Bomke J, Grädler U, Musil D, Buchstaller H-P, et al. Estimation of

1012 Drug-Target Residence Times by $\tau$-Random Acceleration Molecular Dynamics Simulations.

1013 Journal of Chemical Theory and Computation. 2018;14(7):3859-69.

1014 32. Hooley R. Gibberellins: perception, transduction and responses. Plant Molecular Biology

1015 1994. 26: 1529-1555 
1016 33. Liu H, Guo S, Lu M, Zhang Y, Li J, Wang W, et al. Biosynthesis of DHGA12 and its

1017 roles in Arabidopsis seedling establishment. Nature Communications. 2019;10(1):1768.

1018 34. Howe KL, Contreras-Moreira B, De Silva N, Maslen G, Akanni W, Allen J, et al.

1019 Ensembl Genomes 2020 — enabling non-vertebrate genomic research. Nucleic Acids Research.

1020 2020;48(D1):D689-D95.

1021 35. Yamaguchi S. Gibberellin metabolism and its regulation. Annual Review of Plant

1022 Biology. 2008. 59: 225-251

1023 36. Yoshida H, Tanimoto E, Hirai T, Miyanoiri Y, Mitani R, Kawamura M, et al. Evolution 1024 and diversification of the plant gibberellin receptor GID1. Proceedings of the National Academy 1025 of Sciences. 2018 115(33): E7844-E7853.

1026 37. David CC, Singam ERA, Jacobs DJ. JED: a Java Essential Dynamics Program for 1027 comparative analysis of protein trajectories. BMC bioinformatics. 2017;18(1):271-.

1028 38. Wiederstein M, Sippl MJ. ProSA-web: interactive web service for the recognition of 1029 errors in three-dimensional structures of proteins. Nucleic Acids Research.

1030 2007;35(suppl_2):W407-W10.

1031 39. Binenbaum J, Weinstain R, Shani E. Gibberellin Localization and Transport in Plants.

1032 Trends in Plant Science. 2018;23(5):410-21.

1033 40. Salomon-Ferrer R, Case DA, Walker RC. An overview of the Amber biomolecular

1034 simulation package. WIREs Computational Molecular Science. 2013;3(2):198-210.

$1035 \quad 41$ Farmer J, Jacobs D. High throughput nonparametric probability density estimation. PLOS

1036 ONE. 2018;13(5):e0196937. 

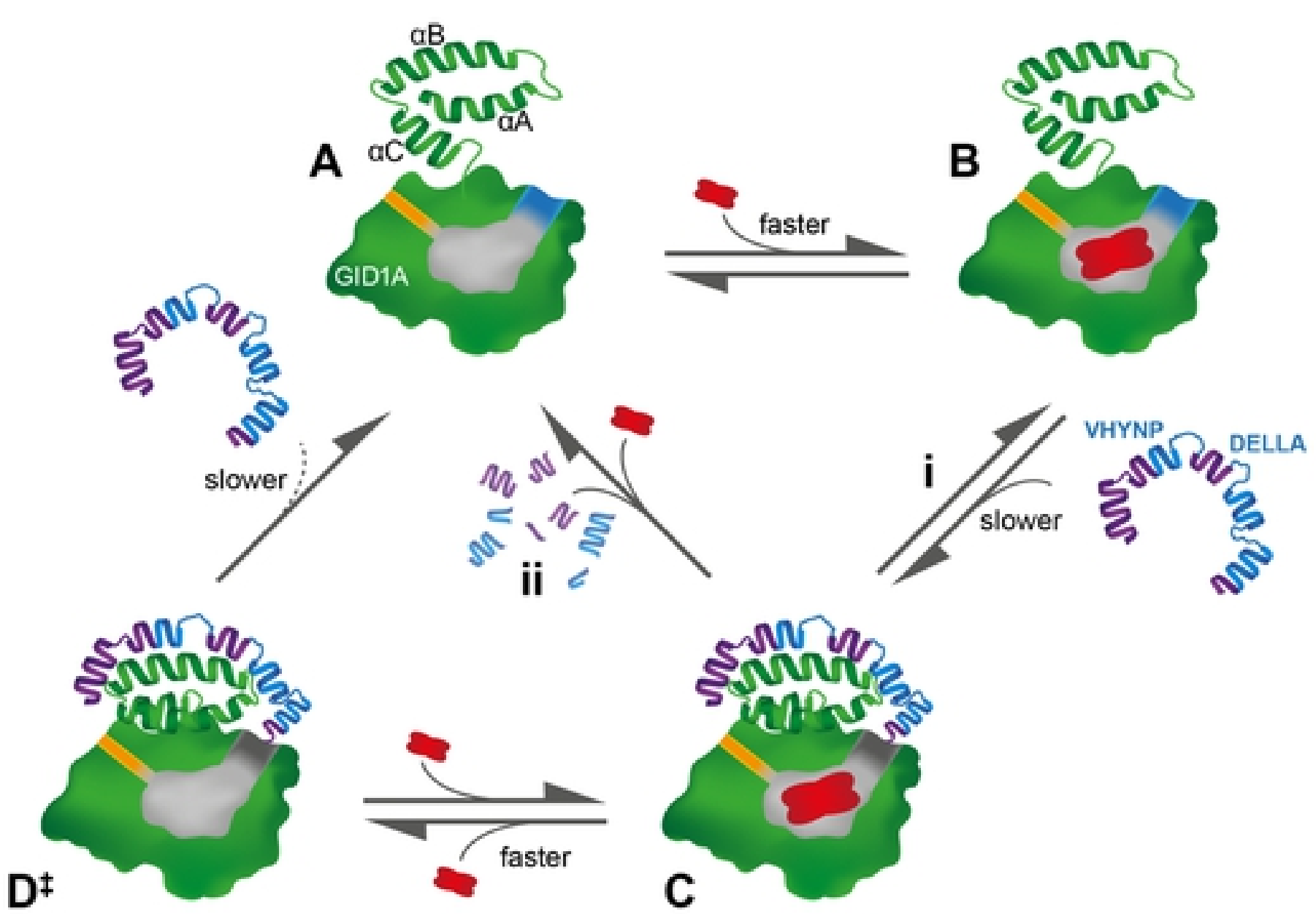

\begin{tabular}{|c|c|c|c|}
\hline Cleft pathway & Channel pattrway & & GAI \\
\hline \multirow{2}{*}{$\begin{array}{l}\text { DELLA-induced Blocked } \\
\text { Cleft pathway }\end{array}$} & Binding pocket & $\begin{array}{l}\text { DELLA } \\
\text { VHYNP }\end{array}$ & $\begin{array}{l}\text { Molecular Recognition } \\
\text { Feature - MoRF }\end{array}$ \\
\hline & Gibberellin & & $\begin{array}{l}\text { Ubiquitin-mediated } \\
\text { proteolysis of GAI }\end{array}$ \\
\hline
\end{tabular}

Figure 1 


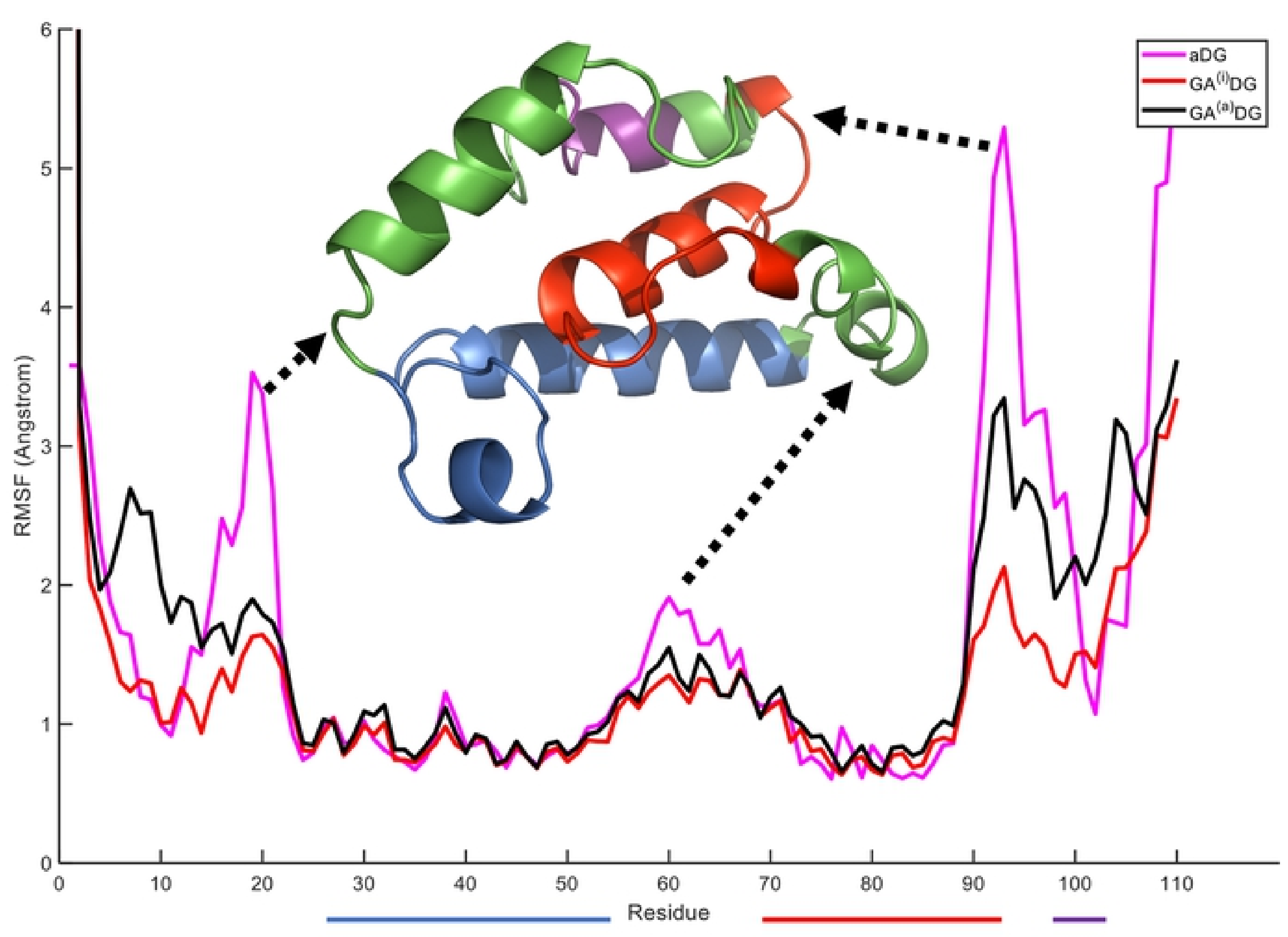

Figure 2 

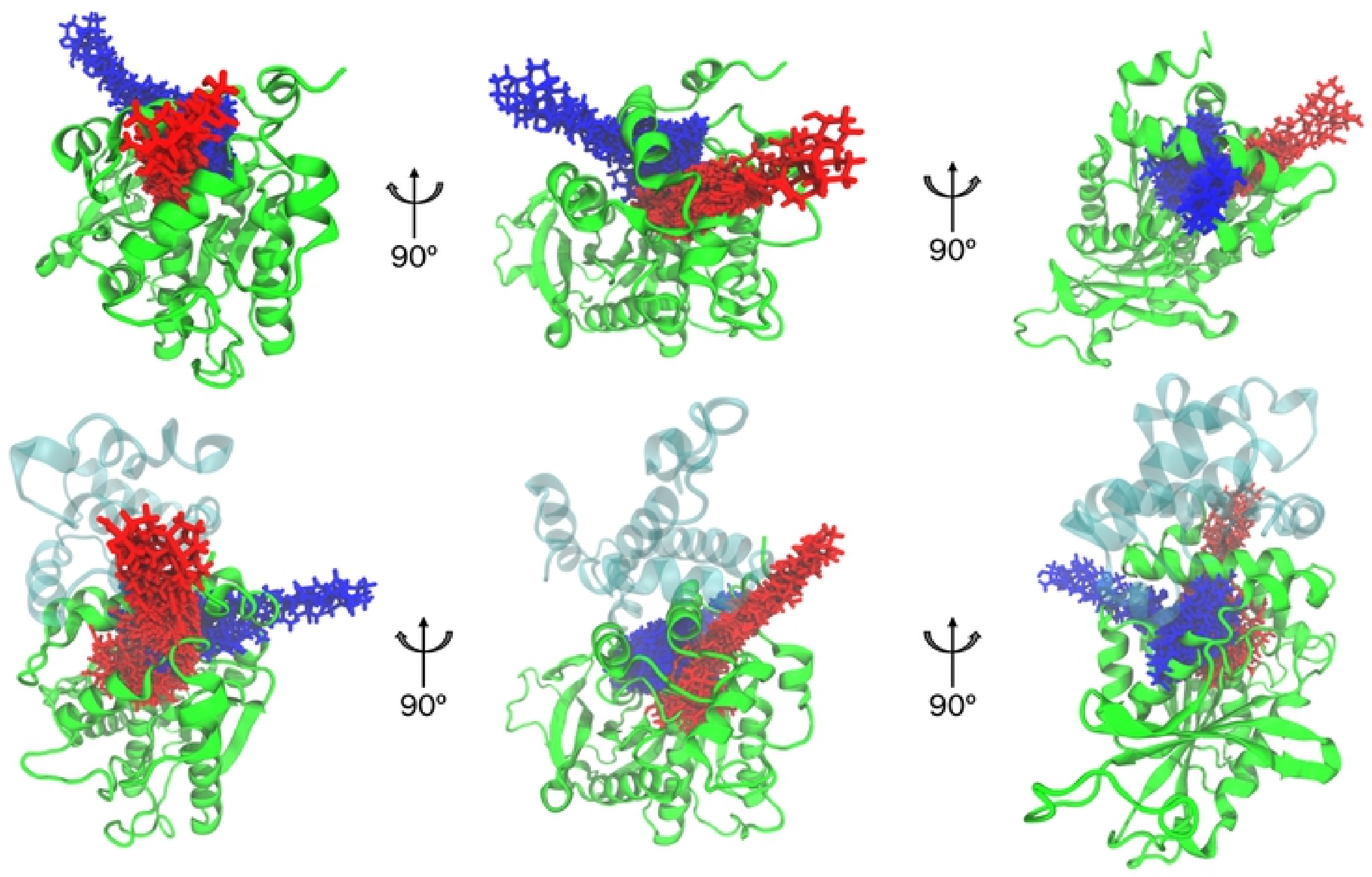

Figure 3 

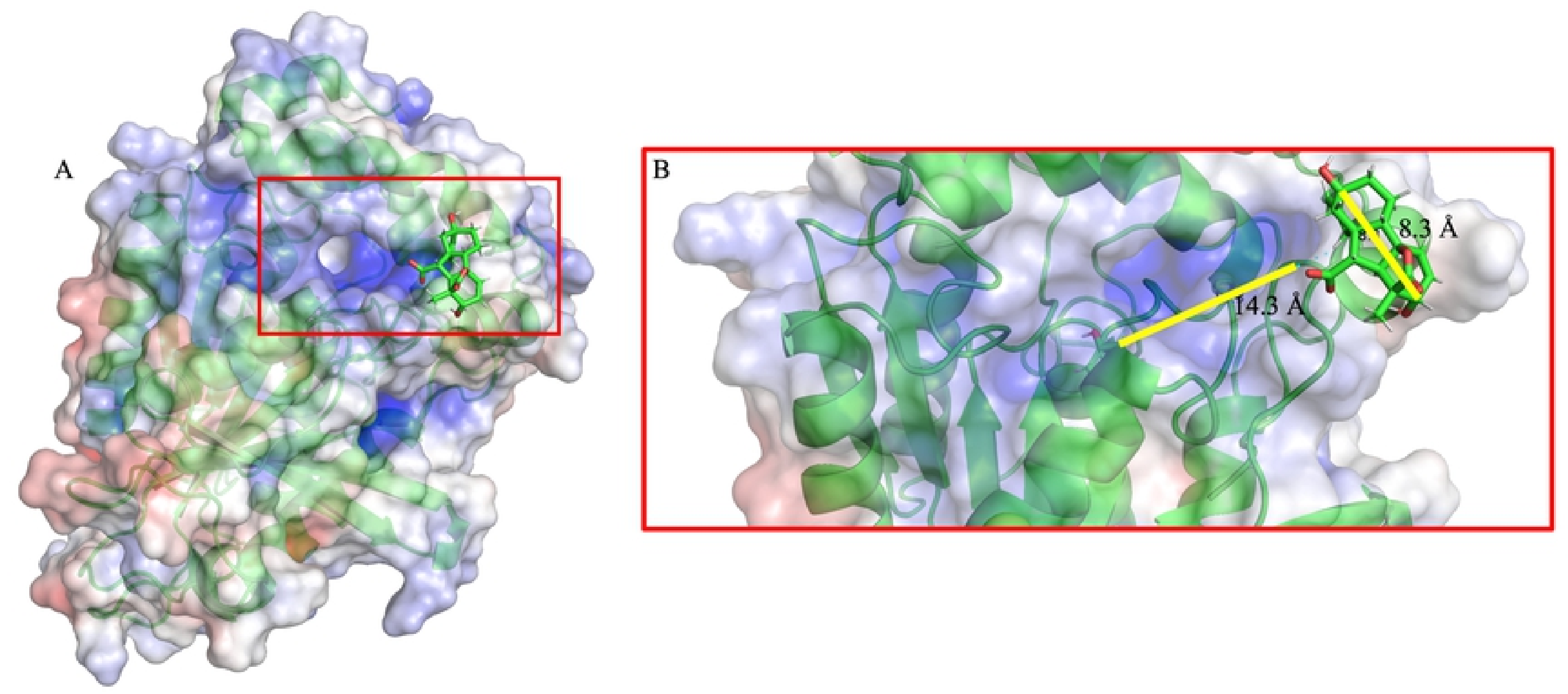

Figure 4 


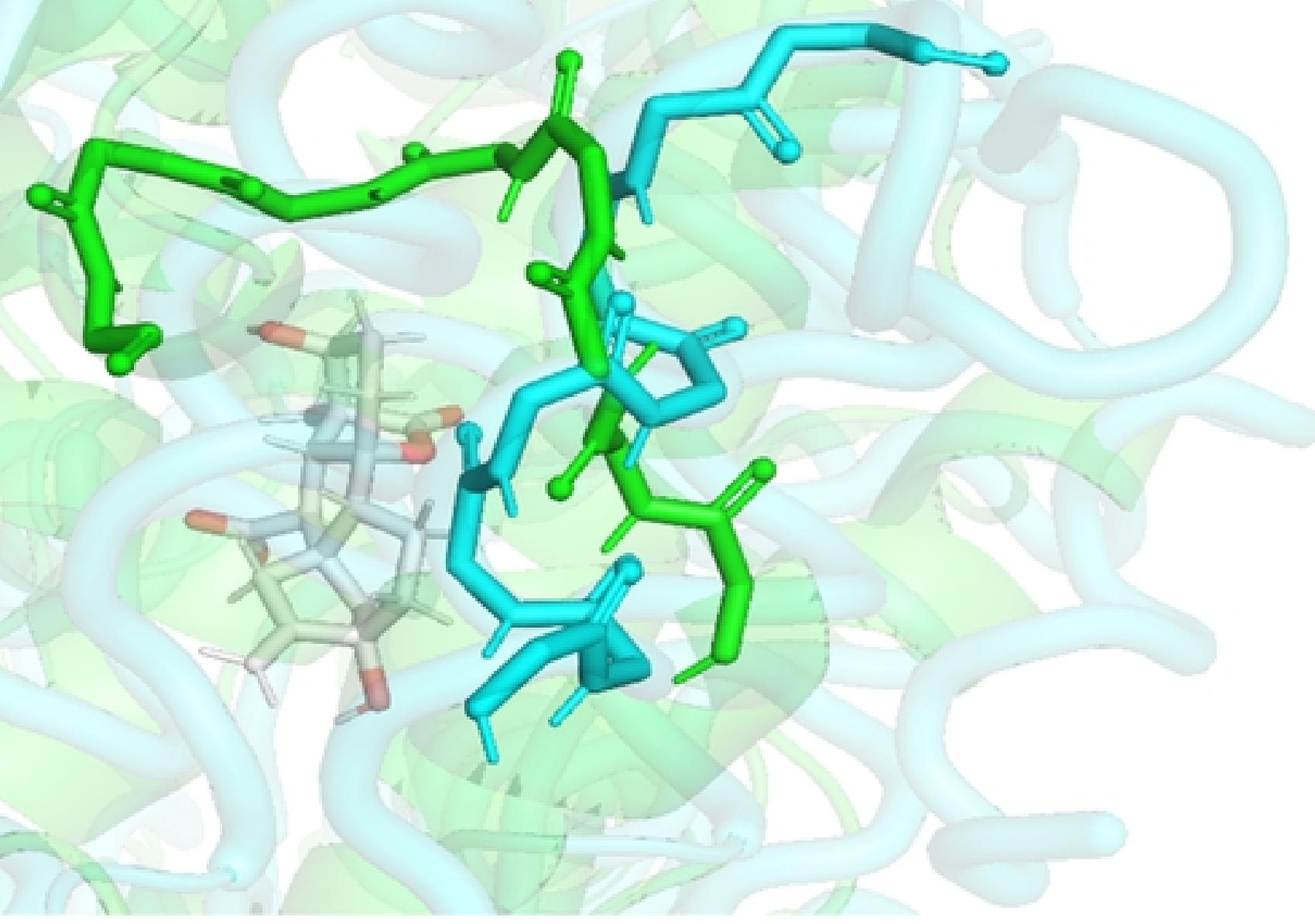

Figure 5 


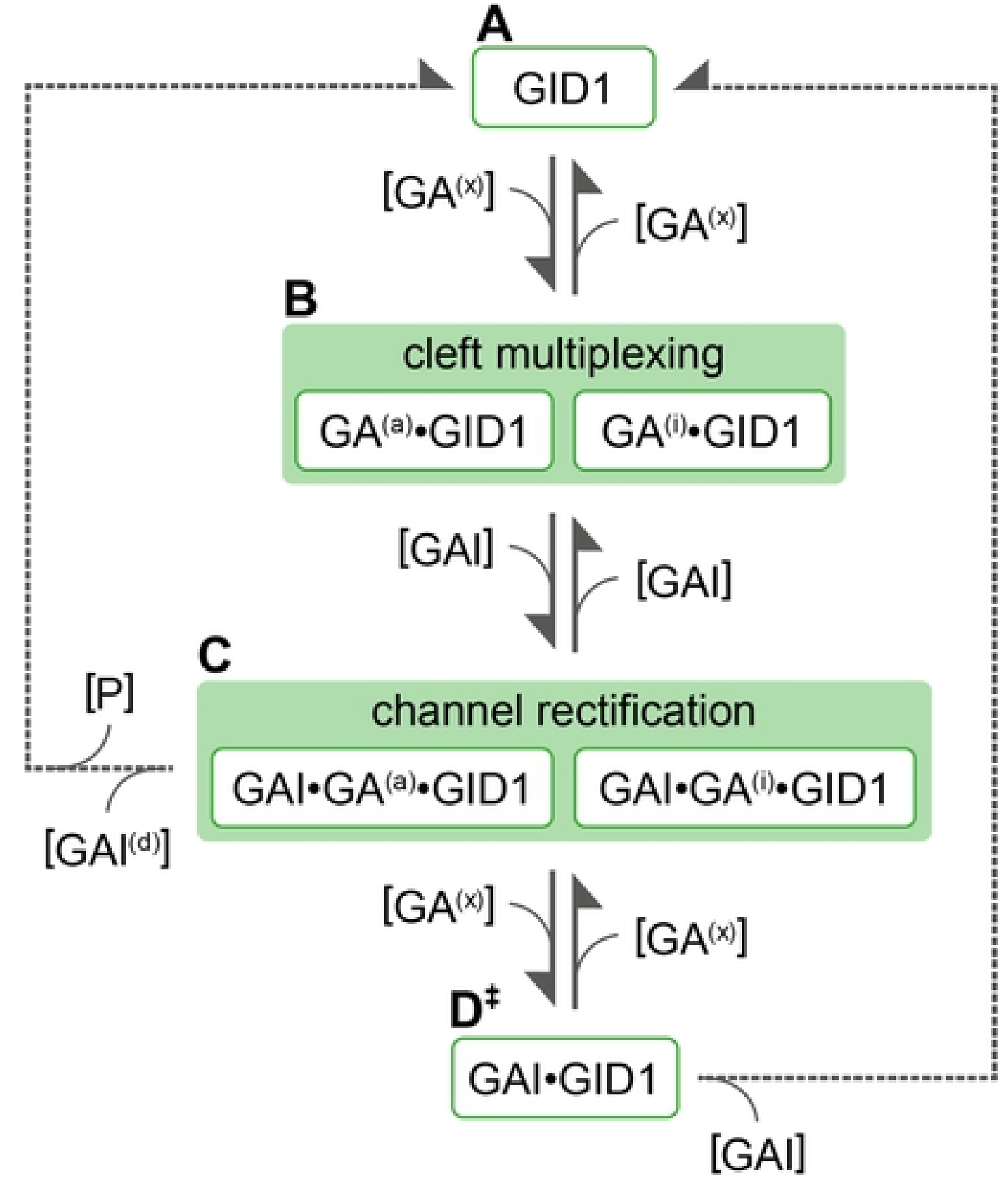

Figure 6 